\title{
Efficient terrestrial laser scan segmentation exploiting data structure
}

\author{
Hamid Mahmoudabadi ${ }^{\mathrm{a}}$, Michael J. Olsen ${ }^{\mathrm{a}}$, Sinisa Todorovic ${ }^{\mathrm{a}}$ \\ ${ }^{a}$ Oregon State University, Corvallis, OR, USA
}

\begin{abstract}
New technologies such as lidar enable the rapid collection of massive datasets to model a 3D scene as a point cloud. However, while hardware technology continues to advance, processing 3D point clouds into informative models remains complex and time consuming. A common approach to increase processing efficiently is to segment the point cloud into smaller sections. This paper proposes a novel approach for point cloud segmentation using computer vision algorithms to analyze panoramic representations of individual laser scans. These panoramas can be quickly created using an inherent neighborhood structure that is established during the scanning process, which scans at fixed angular increments in a cylindrical or spherical coordinate system. In the proposed approach, a selected image segmentation algorithm is applied on several input layers exploiting this angular structure including laser intensity, range, normal vectors, and color information. These segments are then mapped back to the 3D point cloud so that modeling can be completed more efficiently. This approach does not depend on pre-defined mathematical models and consequently setting parameters for them. Unlike common geometrical point cloud segmentation methods, the proposed method employs the colorimetric and intensity data as another source of information. The proposed algorithm is demonstrated on several datasets encompassing variety of scenes and objects. Results show a very high perceptual (visual) level of segmentation and thereby the feasibility of the proposed algorithm. The proposed method is also more efficient compared to Random Sample Consensus (RANSAC), which is a common approach for point cloud segmentation.

Keywords: Terrestrial laser scanning, Segmentation, High Dynamic Range imaging, Computer Vision, Point Cloud, Lidar
\end{abstract}

Preprint submitted to ISPRS Journal of Photogrammetry and Remote SensingMay 26, 2016

(C) 2016. This manuscript version is made available under the Elsevier user license http://www.elsevier.com/open-access/userlicense/1.0/ 


\section{Introduction}

Lidar (Light Detection and Ranging) technology utilizes near-infrared lasers to densely sample objects within view of the scanner in 3D (Vosselman and Maas, 2010), resulting in a point cloud with X, Y, Z coordinates for each sample point. Scanners also encode the strength of the backscatter called laser intensity (i.e., signal degradation as an intensity measurement). Although there are several factors that affect the magnitude of the laser intensity value, this value can characterize reflectance of the target surface (Kaasalainen et al., 2011; Kashani et al., 2015). In addition, most scanners are equipped with digital cameras in order to provide realistic RGB (red, green, blue) color values for each scan point.

It is well accepted that lidar is a powerful tool to produce high resolution, accurate, geospatial information for 3D modeling of an environment. As a result, 3D laser scanning is used in a wide variety of applications including surveying and mapping, industrial plant management, transportation asset management, facilities management, building information modeling, crime scene investigations, coastal erosion, rockfalls, landslides, and seismic displacements, cultural heritage and geologic instigations.

The 3D point clouds and 2D RGB images from a single scan can exceed millions of elemental units; however, these data do not contain semantic meaning (i.e., a point or pixel is not tagged with an object/surface identifier

of what it represents). In many applications, a relatively small portion of the data are actually needed for mapping, modeling, and object extraction. A fundamental step in simplifying data processing and removing redundant information is segmentation (Vosselman and Maas, 2010). In more advanced processes, segmented points can then be classified (e.g., provided with semantic meaning) based on characteristics.

As will be briefly described in the next section, common methods for 3D laser point cloud segmentation require considerable manual labor, using mostly geometric-based approaches with extensive complexity and limited accuracy. Further, many of these procedures tend to fail or require significant manual intervention when faced with real world datasets containing many different kinds of objects and noise. Uneven distribution of points in 3D space and varying scale within the data also present challenges.

A key solution for this problem is utilizing the point cloud structure generated in the scanning process, producing a panorama, which is a $2 \mathrm{D}$ projection of the cylindrical or spherical coordinate system used by the scanner (Olsen 
et al., 2010; Barnea et al., 2007; Barnea and Filin, 2008a,b, 2012). Converting 3D lidar point clouds into these 2D panoramic image maps (PIMPs) enables one to apply available image processing and computer vision algorithms developed for 2D images. There are several algorithms successfully implemented for operating on 2D images (Szeliski, 2010) that have yet to be exploited for use with lidar data. Upon segmentation and analysis, the processed PIMPs then can be easily mapped back into 3D space, resulting in a segmented point cloud.

Measured laser intensity values are a good descriptor for the characteristics of the scanned objects. These values are mainly affected by intrinsic and extrinsic factors of the laser scanners (Kashani et al., 2015). To enhance the quality of the laser intensity PIMP, an empirical correction function is derived and applied on the raw laser intensity values.

To employ the colorimetric information, the proposed approach is applied on both PIMPs of the lidar point clouds and photographic imagery. Digital images are usually captured along with lidar typically contain three layers of information for each point as red, green and blue bands. Although these colorimetric data are generally used for visualization purposes, they have not been substantially used for point cloud segmentation purposes. Certainly, having high quality images that preserve important details can be very helpful to the process. However, a key challenge in digital image acquisition is that the illumination of most scenes vary substantially, resulting in loss of information within underexposed and overexposed regions. The High Dynamic Range (HDR) photography technique is implemented as a solution to cover the full range of light present in the scene and to have detailed digital images in dark and bright areas (Reinhard et al., 2010; Mahmoudabadi, 2015).

The primary objectives of this research can be categorized as follows:

- Develop an approach for intuitive, comprehensive point cloud segmentation in dense, large, 3D terrestrial lidar datasets by applying image processing and computer vision algorithms. Such a method can reduce computation time, improve the perceptual performance of the segmentation in the sense of visual quality, and minimize user intervention,

- Implement HDR photography to improve colorimetric data of digital images and consequently segmentation results; and, 
- Enhance the performance of the segmentation by utilizing an empirical correction formula to correct laser intensity values for range and angle of incidence.

The organization of the paper is as follows: Section 2 provides a brief summary of the current State of the Art. Section 3 describes the methodology. Section 4 shows and discusses the results. Finally, Sections 5 presents conclusions.

\section{State of the Art}

Point cloud segmentation and classification research is rapidly progressing for a wide variety of applications. Many novel approaches were developed for Aerial Laser Scanning (ALS), Mobile Laser Scanning (MLS), and range camera derived point clouds, which we will only briefly summarize herein as relevant. Rather, we focus this review on techniques that were developed for terrestrial laser scanning (TLS).

\subsection{Geometry}

Current algorithms in the literature chiefly focus on segmenting laser scanner point clouds (typically from airborne platforms) into planar regions or ground points. These are logically the most common due to the easy determination, modeling, and frequency of planar surfaces in urban environments. However, because these approaches operate in 3D space, they are computationally costly and often require the use of finely-tuned parameters, which can be laborious when applied to broader and larger datasets. There are also a variety of geometric methods for ground-filtering and vegetation removal (see Vosselman and Maas (2010) for an overview of approaches). These procedures typically operate using the fundamental algorithms of Random Sample Consensus (RANSAC) (Fischler and Bolles, 1981), Hough transforms, surface or region growing (based on proximity, slope, curvature, and surface normal) from a seed location (Rabbani et al., 2006), finding discontinuities (Wang and Shan, 2009) in the point cloud, a k-means clustering approach (Chehata et al., 2008), voxelation (Douillard et al., 2011) or using fuzzy parameters in relative height differentials (Biosca and Lerma, 2008). The RANSAC algorithm randomly and iteratively selects minimal sets (smallest number of samples required to uniquely define a model) to find the optimal parameters to fit a candidate mathematical model. Then, the parameters are evaluated 
to determine the consensus for the point set for the best fit to the model. It is a fairly robust approach to outliers.

In $\mathrm{Pu}$ et al. (2006) a simple planar surface growing algorithm is used to extract building features (walls, windows, and doors) from terrestrial laser scanned data using properties such as size, position, direction, and topology for each planar segment. Moussa and El-Sheimy (2010) consider the size of the patch to distinguish between buildings and vegetation. Lin and You (2006) first compute the normal vector for each point by tensor-voting, followed by classification through a cluster density method based on similar normal vector directions.

Gorte (2007) presents a plane segmentation approach for terrestrial lasers scans that works directly on a range image representation of the scan. Calculating image gradients as the rate of change in the distance that is observed between adjacent pixels (which represent measurements) helps characterize a plane in 3D space. Regions of adjacent pixels with similar gradients are grouped into same segment by a region-growing image segmentation.

Although primarily developed for robotics, the Point Cloud Library (PCL) (Rusu and Cousins, 2011) is a recent, powerful, open source library for segmentation through extraction of geometric primitives (planes, cylinders), laser intensity, normal vectors as well as raw RGB color, if available.

Serna and Marcotegui (2014) segment connected objects on a created digital terrain model (DTM) from 3D point clouds. Next, a support vector machine (SVM) classifies the objects based on several geometrical and contextual features. Finally, the label and class images are reprojected to the 3D point cloud. The approach was tested on benchmark MLS and ALS databases from Paris (France) and Ohio (USA). Weinmann et al. (2015) introduce a novel framework involving four successive components as (i) neighborhood selection, (ii) feature extraction, (iii) feature selection, and (iv) classification.

The aforementioned approaches have shown successful results. Nonetheless, challenges arise when using these approaches with TLS data. First, a scale parameter is required for neighborhood selection, which can vary significantly throughout a TLS point cloud due to substantial point density differences. Second, these methods only consider geometric information and do not consider intensity and photographic information. Third, supervised classification requires training features, which can be prone to bias and needs a balanced distribution of training examples per class for training process, which may not be realistic in a scene. Finally, many algorithms have been developed for small datasets ( $<5$ million points) and have difficulty scal- 
ing up to extract objects of interested in larger datasets. With current TLS systems, it is common to have more than 20 million points per each scan.

\subsection{Colorimetric data}

The recent availability of high-resolution digital cameras and laser scanners has given rise to extremely rich colored geometric datasets. Many techniques to combine the laser scanning dataset with the digital images are presented mostly in heritage documentation (Douillard et al., 2011; Caroti and Piemonte, 2007; Kadobayashi et al., 2004). Unfortunately, even a colored point cloud is far from informative on its own. To further utilize the sample information, we must first segment and categorize the points in the cloud into objects of interest. In Strom et al. (2010), segment unions are proposed as an extension of graph-theoretic segmentation followed by a dynamic segment union criterion, based on both color and surface normals. Triebel et al. (2005) use a hierarchical variant of the popular expectation maximization algorithm to determine an optimal set of planes that fit the data.

The Yu et al. (2011) algorithm has three steps. In the first step, ground detection by establishing upper thresholds for both absolute offset from local minima and absolute differential change for enforcing smoothness. In the second step, the mean shift segmentation algorithm is used to divide and conquer the remaining objects. Finally, they use a manifold embedded mode seeking method similar to mean shift to segment the cloud into objects. Sok and Adams (2010) also used a mean shift smoothing algorithm to segment the image into clusters. Then these clusters are used to group the corresponding 3D point cloud to imagery data. Finally, Principal Component Analysis (PCA) is applied to each group to categorize them as planar, linear, or random. They concluded that applying PCA to combination of a imagery and 3D point clouds is more robust to extract planes compared to the naive application of PCA. Cheng et al. (2011) combine multi-view aerial imagery and lidar data to reconstruct 3D buildings.

Another approach that combines lidar and digital images to generate a 7-dimensional hyperspace (X, Y, Z, Red, Green, Blue, intensity) is presented in (Mahmoudabadi et al., 2013). In this approach, first, a superpixel algorithm (Simple Linear Iterative Clustering) is used to cluster the image pixels based on color and their position, followed by determining the normal vector of each projected superpixel. A rigid mathematical classifier and a multiclass support vector machine are performed for categorization of superpixels and object extraction based on the generated normal vectors. Finally, the 
misclassification of each superpixel is refined by using k-nearest neighbor algorithm on its immediate neighbors.

\subsection{Laser intensity}

In Niemeyer et al. (2012) a Conditional Random Field (CRF) algorithm is applied to classify five object classes (building, low vegetation, tree, natural ground, and asphalt) using geometrical features and intensity values of airborne lidar data. In a CRF, each node as well as each edge is associated to a feature vector. Finally, a conversion from $3 \mathrm{D}$ point cloud to $2 \mathrm{D}$ objects is performed for each class of interest.

Mallet et al. (2011) classified 3D point clouds in urban areas, using fullwaveform data representing three main classes: building, ground, and vegetation. The combination of 3D point cloud and full waveform features allowed them to create a 27-component feature vector, including 19 from the point cloud and 8 from waveform data. The next step is a feature selection that claimed three full-waveform features, namely the amplitude, the cross-section per illuminated area, and the backscatter coefficient. Finally, a support vector machine (SVM) classifier is used to label the point clouds. Additionally, they showed that the intensity amplitude correction and calibration procedure is fundamental in order to extract discriminant features.

Kashani et al. (2015) reviewed effective parameters on intensity measurements, basic theory, and several laser intensity correction methods. As an example, Höfle and Pfeifer (2007) present two independent methods for correcting ALS intensity values. The first is a mathematical model that performs a least squares adjustment to estimate the best parameters for a given empirical correction function, including intensity and range. The second is a theoretical correction, which is based on the radar equation and depends on information about the atmospheric conditions during data acquisition. The authors highlight the great potential that corrected laser return intensity has as an independent and additional variable for segmentation and classification analyses.

\subsection{Structure}

The angular structure of point cloud data in terrestrial laser scans can be taken advantage of for point cloud segmentation. In (Zeibak and Filin, 2009; Barnea and Filin, 2012, 2008b,a; Olsen et al., 2010), projecting the $3 \mathrm{D}$ data to a $2 \mathrm{D}$ panoramic grids shows significant potential to simplify the segmentation process. (Zeibak and Filin, 2009) uses the panorama to identify 
objects appearing in different shapes, sizes, range and locations within the scene and relatively to the scanner. Generally, point clouds are segmented based on smoothed surface normal and then points are classified as objects using the derived direction and applying the dot product based analysis. In (Barnea and Filin, 2008b) three datasets were created using the intensity value in the range panorama, the surface normals, and true color channels. The mean-shift algorithm is used as the segmentation scheme for each cue individually. For the normal based cue the ground and the facades appear as complete segments and for the color channel some of the facades and vehicles were extracted partially. Finally, an integration approach is applied across all data sets. Barnea and Filin (Barnea et al., 2007; Barnea and Filin, 2008a, 2012, 2008b) focused this effort to extract trees in the point cloud. For each of the channels (range, intensity, Luv and HSV color), they determined that the range and intensity values provided the highest descriptive power for tree points.

\section{Methodology}

The proposed approach has four main steps (Figure 1): preparation, segmentation, integration, and merging. The following sections describe each step.

\subsection{Scanned data to PIMPs (Panoramic Image Maps)}

Terrestrial laser scanners operate on a spherical or cylindrical structured format with measurements separated by a fixed angular offset as opposed to a final distance sampling resolution on a target surface. For each measured point, its distance to the laser scanner, namely the range $(\rho)$, the horizontal $(\phi)$ and the vertical $(\theta)$ angles are recorded. Although laser scanners natively sample in angular increments, the output is often provided as ( $\mathrm{x}$ y $\mathrm{z}$ ) coordinates.

To facilitate the processing and establish a gridded neighborhood, the 3D lidar point cloud is treated as a 2D panoramic image map (PIMP) representation whose axes are the horizontal $(\phi)$ and the vertical $(\theta)$ scanning angles, and the measurements are the intensity values. Because of the fixed angular spacing (defined by system specifications), a lossless angular structured file can be set. The Leica PTX format, which is an ASCII based format for point cloud data retains the structure, colorimetric and laser intensity values from the original scan plus additional registration information (Leica, 2014). The 


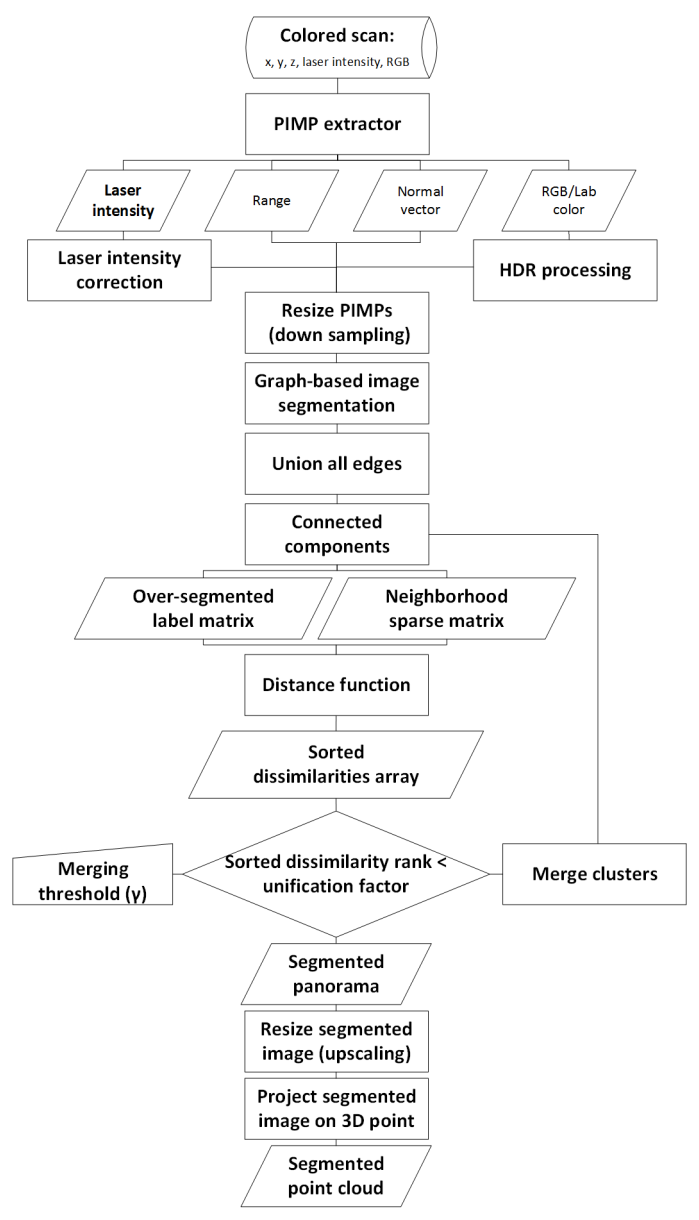

Figure 1: Flowchart highlighting key steps of implementing the proposed segmentation approach.

ASTM E57 format also preserves the angular structured format of the scans. E57 is not only capable of storing point cloud data from laser scanners and other 3D imaging systems, but it also stores associated 2D imagery and core meta-data (Huber, 2011). Other formats such as ASPRS LAS do not directly preserve this data structure.

The process of extracting the PIMPs of the interest parameter from the scan data simply requires exploiting the angular structured format of the scan and storing the values in 2D array files. An adapted version of the code developed by (Olsen et al., 2010) reads information from a structured lidar file (e.g., PTX format) and to export all input channels into PIMPs. 
Pixels representing pulses with no-return areas in the scene (e.g., the sky) are set as transparent so they are not included in the analysis. PIMPs values are typically $0-255$, but multiple channels can be used (e.g., RGB) to store information with higher precision. The following PIMPs are extracted from acquired scans and are shown in Figure 2:
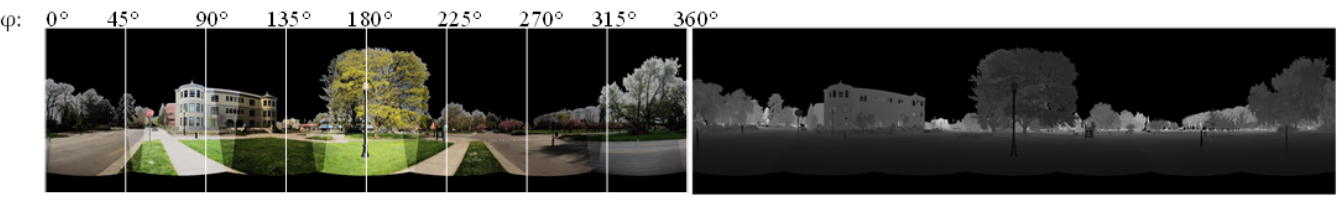

A) RGB

B) $\rho$
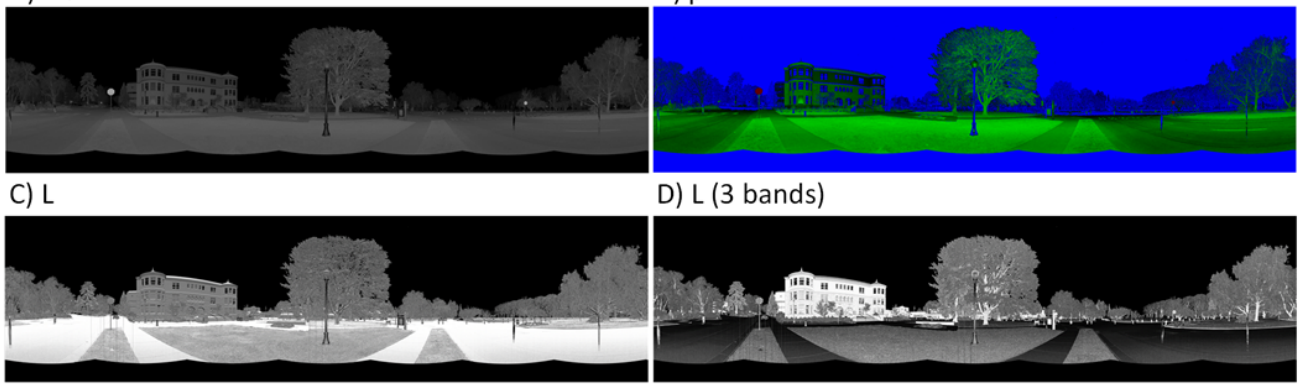

D) L (3 bands)
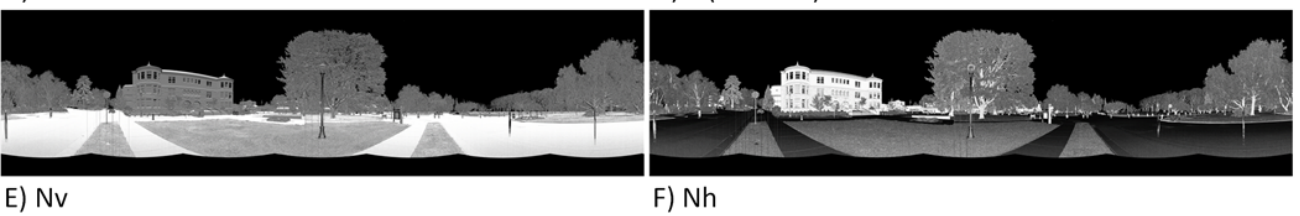

E) $\mathrm{NV}$

F) $\mathrm{Nh}$
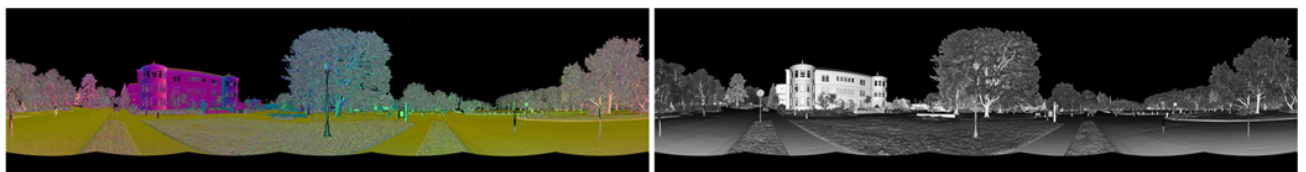

G) Nxyz

H) $\beta$

Figure 2: Generated PIMPs A) $R G B$ - colors from single shot digital images B) $\rho$ - range C) $L$ - laser intensity in grayscale D) $L_{3 b a n d}$-laser intensity spread in RGB bands E) $N_{v}$ vertical normal F) $N_{h}$ - horizontal normal G) $N_{x} y z$ - normal vector spread in RGB bands H) $\beta$ - incidence angle.

\section{RGB color}

Digital cameras are widely used to color the laser point cloud. The integration of camera and laser scanner is based on different coordinate systems and their relative transformation matrices. The camera is calibrated with the laser scanner so that the offset (translation and rotation) between the camera origin and scanner origin can be accounted for. Then the captured images can be mapped to the point cloud (Riegl, 2009; Leica, 2014). The transformation between the camera and laser scanner is described in detail in (Barnea and Filin, 2008a, 2012). The RGB values are assigned to the 
3D points using this transformation matrix. These colorimetric values are computed directly by mapping the images to the laser scan point cloud and can be performed with minimal effort in most manufacturer software.

\section{Laser intensity}

Almost all laser scanners record the strength of returned laser signal as laser intensity (L) for each point, which can be mapped in the PIMP. While the approach to map the intensity values in (Olsen et al., 2010; Barnea et al., 2007; Barnea and Filin, 2008a, 2012, 2008b) is to scale the values to the range

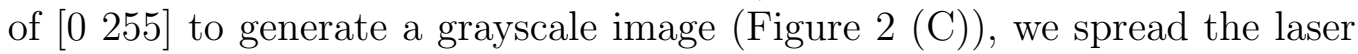
intensity values across three bands using statistical parameters such as the average $\left(\mu_{L}\right)$ and standard deviation $\left(\sigma_{L}\right)$ (Figure $2(\mathrm{D})$ ). The laser intensity values are assigned to RGB bands based on the following rules:

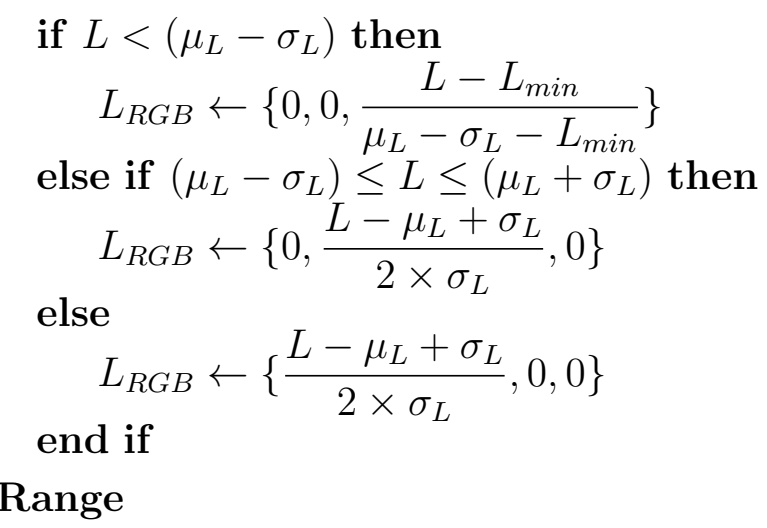

Ironically, although the range is one of the basic measurements of the scanner and determined based on the time-of flight, most output files from the scanner do not contain range values. Fortunately, the range values can be easily re-calculated using the Euclidian distance from the scanner (Origin $0,0,0)$ to the point coordinate values $(\mathrm{X}, \mathrm{Y}, \mathrm{Z})$. Figure $2(\mathrm{~B})$ shows the range PIMP as 8 bit grayscale image.

\section{Normal Vectors}

The normal vector at each pixel is defined as a line or vector that is perpendicular to the given pixel based on a surface formed by its neighboring pixels. The normal vector of the surface from which the point was acquired can be computed by using neighboring points found using the scan angular structure. 
To calculate a normal vector at a pixel with a laser intensity value, there must be at least two immediate neighbor points with laser intensity values (i.e., not empty pixels where a lidar return was not obtained). Then, the gradient between the current pixel and its neighbors are calculated $(\triangle b a, \triangle c a, \triangle d a)$. Finally, the normal vector for the current pixel is computed as the average of all cross products for each gradient pair.

Barnea and Filin $(2012,2008 \mathrm{~b})$ represented normal vectors by mapping each component of the normal vector $\left(N_{x}, N_{y}\right.$, and $\left.N_{z}\right)$ from a value range of -1 to 1 to a value range of 0-255 in the R, G,B channels, respectively (Figure $2(\mathrm{G}))$. A limitation of assigning each component of the normal vector with an individual color band is that objects with multiple sides facing different directions are colored differently and divided into several parts. To reconcile this information, additional PIMPs were created to represent the vertical $\left(N_{z}\right)$ and horizontal $\left(N_{h}\right)$ component as:

$$
N_{h}=\sqrt{N_{x}^{2}+N_{y}^{2}}
$$

\section{Angle of incidence}

The angle of incidence can be defined as the angle between incoming laser pulse and the normal vector at each sampled point. The incoming laser pulse $(\vec{\rho})$, can be represented as a vector whose components are the coordinates of the points in the scanner coordinate system $(\mathrm{x}, \mathrm{y}, \mathrm{z})$ since the scanner origin is at $(0,0,0)$. Using this vector $(\vec{\rho})$ and the normal vector $(\vec{N})$ of the surface, it is possible to calculate the angle of incidence $(\beta)$ as follows:

$$
\operatorname{Cos}(\beta)=\frac{\vec{\rho} \cdot \vec{N}}{\|\rho\| *\|N\|}
$$

For the surfaces that are perpendicular to the direction of the scanning laser ray, the angle of incidence is close to zero and for surfaces whose normal vector is perpendicular to the incoming laser pulse (e.g., parallel surfaces), the angle of incidence is larger, up to 90 degrees. Because $\cos (\beta)$ is always in the range of $[01]$, the grayscale image can be generated using $\cos (\beta)$ as representative of the incidence angle (Figure $2(\mathrm{H})$ ).

\subsection{Data improvement}

In this paper two solutions are suggested to enhance the input data and create auxiliary data. The first method is for colorimetric RGB data by 
applying HDR imaging and the second method is an empirical function for laser intensity correction.

\subsubsection{High Dynamic Range images}

While imagery can be easily mapped to the point cloud, variable lighting conditions create several problems that often result in color information loss or degradation in the photographs. First, the single-exposure digital images contain underexposed and overexposed regions without colorimetric information. Second, the single exposure photographs more often lead to inconsistencies between adjacent images due to the illuminance variety in the scene. Digital images with improved detail in dark and bright areas can be generated using the HDR photography technique (Reinhard et al., 2010). In the HDR method, a sequence of photographs from the same viewpoint with different exposures is combined to improve coverage of the wide range of luminance levels found in natural and artificial scenes.

Because the pixel values in raw HDR images are above the dynamic range of an ordinary display device, a tone mapping operator is used to shrink the color values in the same range as other panoramas and possibly to display. The tone mapping operator selected for this study was a recently developed method (Mahmoudabadi, 2015) which proved to be a general purpose operator that works well on a wide variety of scene types and under a wide variety of conditions. This technique utilizes geostatistical variography to describe spatial dependencies between luminance values. Then the parameters of the variogram model produce optimal luminance across the image while still preserving local details in the bright and dark regions of the image. Although tone mapping the HDR is not necessarily for display, tone mapped HDR can make the segmentation process more straightforward.

\subsubsection{Laser Intensity correction}

The laser radar range (lidar range) equation is used to relate the received optical power to many other parameters such as the transmitted power, system characteristics, acquisition geometry, environmental parameters, and surface attributes of the scanned target (Shan and Toth, 2008). Laser scanner manufactures apply different forms of the equation and select different point(s) on the return waveform for the laser intensity measurements (Kashani et al., 2015). 


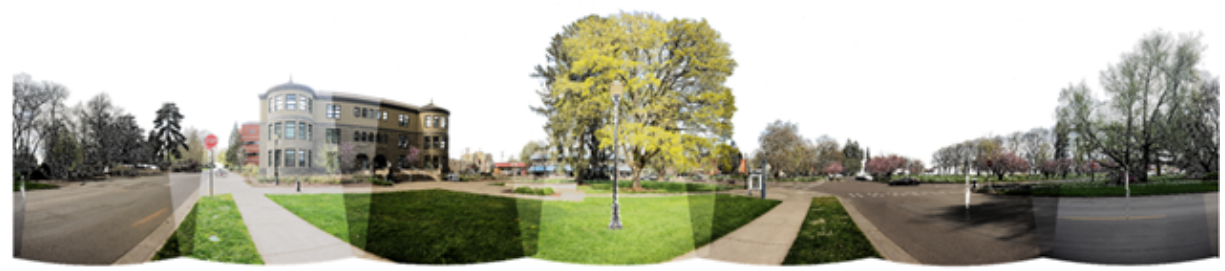

A) RGB PIMP

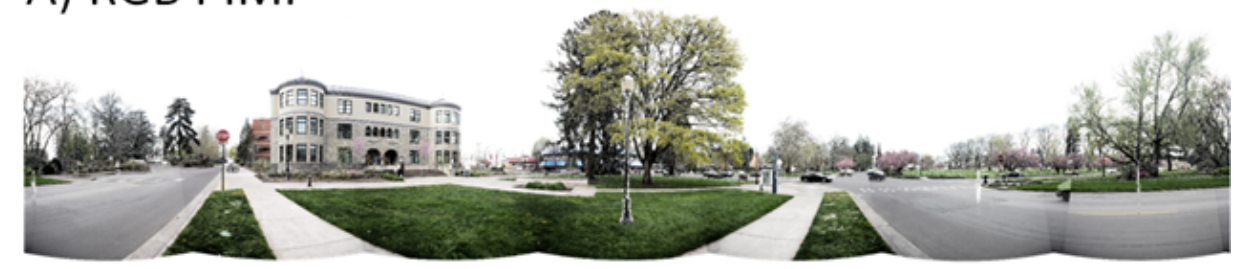

B) HDR PIMP

Figure 3: Created PIMPs from A) single shot images in automatic mode and B) HDR imaging. Inconsistencies between adjacent images and loss of detailed information in regions with extreme lighting in single shot images are obvious.

While numerous studies for airborne laser scanning (ALS) intensity correction have been completed recently (Ding et al., 2013; Briese et al., 2012; Vain et al., 2009), relatively little research has been conducted for terrestrial laser scanning (TLS) intensity correction. In Kaasalainen et al. (2009, 2011); Pfeifer et al. (2008) and Kashani et al. (2015) it is shown that the intensity of the return signal received by a lidar detector is mainly affected by instrument factors, the target scattering features, and the acquisition geometry of the instrument relative to the surface. Range and angle of incidence tend to have the largest influence on relative intensity values when compared with other factors.

Because the laser intensity values provided by the manufacturer in their native file format are typically scaled to a specific range of values (e.g., 0-1, $0-255$, or $0-65,535)$, and the processes used for scaling the sensor voltages and any adjustments applied are often unknown, using an empirical correction method is a solution to adjust the intensity values. Applying an empirical correction method means recalculating the laser intensity values by a function of effective variables (e.g., range and incidence angle) (Ding et al., 2013; Jutzi and Gross, 2009). Empirical methods are suitable when physical and sensor-related parameters in the lidar range equation are unknown. To find the empirical methods, scanning homogeneous surfaces with varying parame- 
ters (e.g., varying ranges and incidence angles) is required. In this study, four homogeneous surfaces are selected: building, grass, cars, and cement. The observation of the laser scanning data of these surfaces showed a nonlinear relationship between laser intensity, range, and incidence angle (gray dots in Figure 4). Range and incidence angles are relative changes that are often expressed in terms of ratios in many papers. One problem with ratios is their lack of symmetry; which means inverting a ratio with value in the range 1 to infinity results in a ratio in the range of 0 to 1 (Dallal et al., 1999). Because the goal of modifying laser intensity values is to be able to identify different surfaces regardless of the changes in range and angle of incidence, a logarithmic empirical formula is a good solution; logged ratios restore symmetry and linearize the non-linear relationships between parameters. Another feature of the logarithm, which makes it so useful in analyzing data, is that it reduces big values while stretching out small values and compresses the upper and lower end of the distribution. So the proposed empirical correction function is introduced as follows:

$$
L_{c}=\frac{\log \left(\frac{L \times \rho}{\cos (\beta)+1}\right)}{\log \left(\frac{L}{\cos (\beta)+1}\right)+L}
$$

where $L$ is the basic recorded laser intensity value (raw intensity) scaled in the range of (0-255), $\rho$ is a normalized range value as described in the previous section ranged in 8 bit (0-255), and $L_{c}$ is the dimensionless corrected laser intensity value.

This formula corrects for the acquisition geometry, reduces the variability of the laser intensities for a specific object, and remains consistent across different range and incidence values, enabling it to represent reflectance characteristics of the material rather than signal degradation from scanning geometry.

The behavior of the correction formula on the selected homogeneous surfaces is shown in Figure 4. The laser intensity values degrade with increasing range and incidence angle. Applying the proposed formula can correct for these degradation such that intensity values are more representative of the surface material. This is evidenced by the fact that the standard deviations are significantly reduced when compared with the uncorrected intensity values for each material (Table 1). 

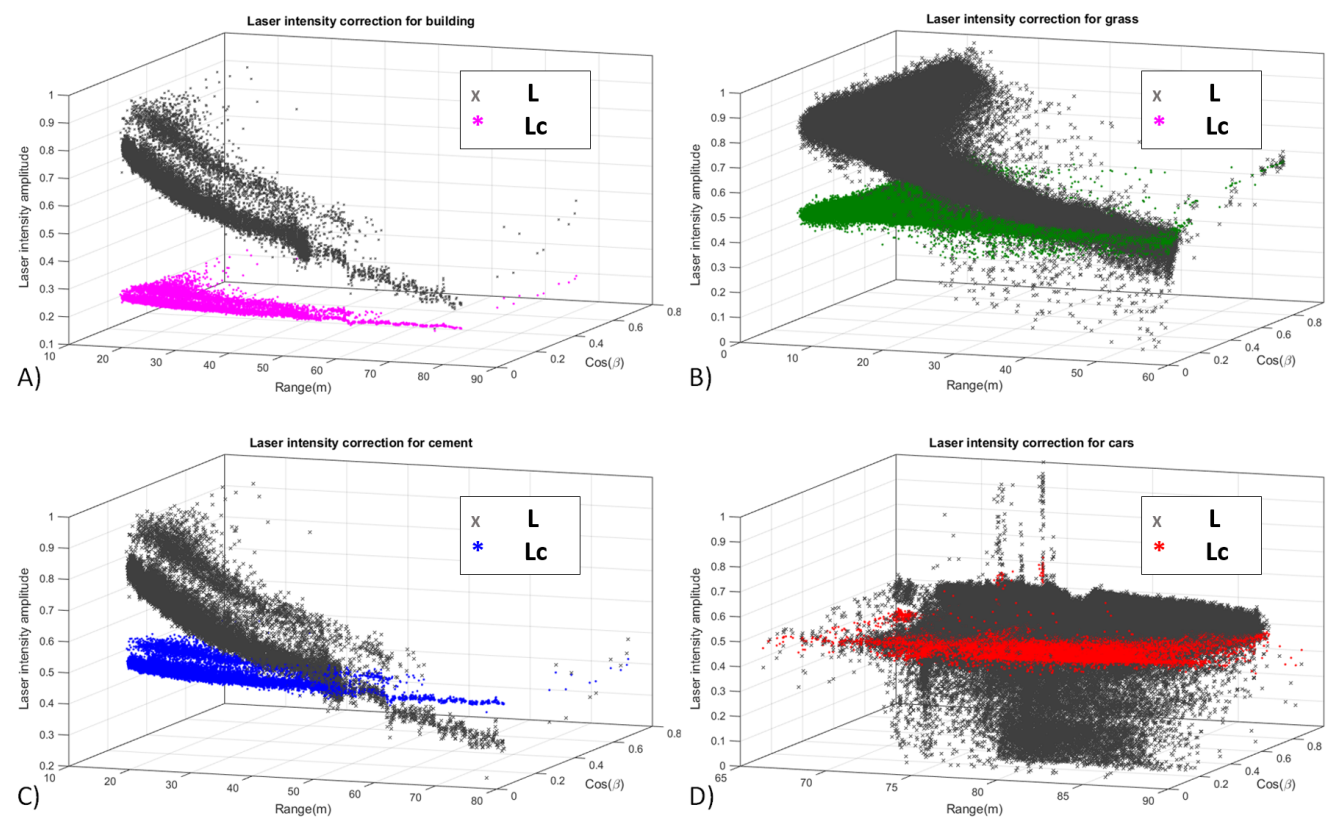

Figure 4: The performance of the laser intensity correction formula on laser intensity values of different materials A) building, B) grass, C) concrete, D) cars

\subsection{Segmentation}

Segmentation is often the first step in extracting information from a point cloud for high level processing. A segmentation algorithm groups point clouds that belong together according to some similarity measurement. The transformation of the data into a PIMP enables the use of computer vision-based image segmentation procedures for segmenting the lidar point cloud.

Because the generated PIMPs from scans usually have very large image dimension for high resolution scans (e.g., $7219 \times 2003$ pixels for scan with a $0.05^{\circ}$ fixed sampling increment), applying some image segmentation algorithms could require significant computation time. A solution to reduce computation time is to scale the PIMPs down to a typical image size (e.g., less than 1000 pixels). Among the many available interpolation methods for image rescaling, the bicubic method (Szeliski, 2010), which calculates the output pixel value as weighted average of pixels in the nearest 4-by-4 neighborhood is preferred. The images can then be rescaled to their original sizes after the image segmentation algorithms are completed. Because this downsampling is a trade-off between the computational time and the quality of 
Table 1: Average and standard deviation of intensity values for the selected materials before and after applying laser intensity correction

\begin{tabular}{l|cccc} 
& $\mu_{L}$ & $\mu_{L_{c}}$ & $\sigma_{L}$ & $\sigma_{L_{c}}$ \\
\hline building & 0.4260 & 0.3020 & 0.0734 & 0.0275 \\
grass & 0.7577 & 0.5040 & 0.0981 & 0.0157 \\
concrete & 0.7003 & 0.5061 & 0.1067 & 0.0205 \\
cars & 0.6097 & 0.5394 & 0.0502 & 0.0422
\end{tabular}

the results, the presented experiments in the Section 4 are calculated without down-sampling.

While there are numerous image segmentation algorithms available in the literature (Szeliski, 2010), we utilized a graph-based image segmentation (Felzenszwalb and Huttenlocher, 2004), which is one of the most common techniques. In graph-based techniques, each pixel represents a node and the difference between two pixels is defined as the edge between those pixels. Graph-based image segmentation is based on selecting edges from a graph, where each pixel corresponds to a node in the graph, and certain neighboring pixels are connected by undirected edges. After defining a predicate for measuring the evidence for a boundary between two regions using a graph based representation of the image, a segmentation algorithm that makes greedy decisions is applied for segmentations that satisfy global properties. There is one runtime parameter for this algorithm, which is a threshold value $(\tau)$ that effectively sets a scale of observation. A larger $\tau$ causes a performance for larger components which results in larger clusters. The algorithm runs in nearly linear time $O(n \log n)$ with the number of graph edges (pixels) and is fast in practice (Felzenszwalb and Huttenlocher, 2004). The output of the graph based image segmentation is a label matrix with the same dimensions as the input PIMPs, where different areas are labeled with a different number.

Figure 5 illustrates the results of the graph based segmentation on several example PIMPs of quad outside Memorial Union in Oregon State University (MU). This scene contains buildings at far distances that are partially occluded by trees and many patches of grass and concrete pavements as ground. As each PIMP describes a specific characteristic of the scene, there are some false results in segmentation on each individual PIMP. For example, $N_{h}$ and $N_{v}$ PIMPs are good descriptors to recognize vertical and horizontal planes in the scene but they can not distinguish between different materials of ground 
(e.g., concrete pavement and grass patches). Further, the range PIMP shows striped artifacts while the RGB PIMP has some false segments due to inconsistencies between adjacent images.

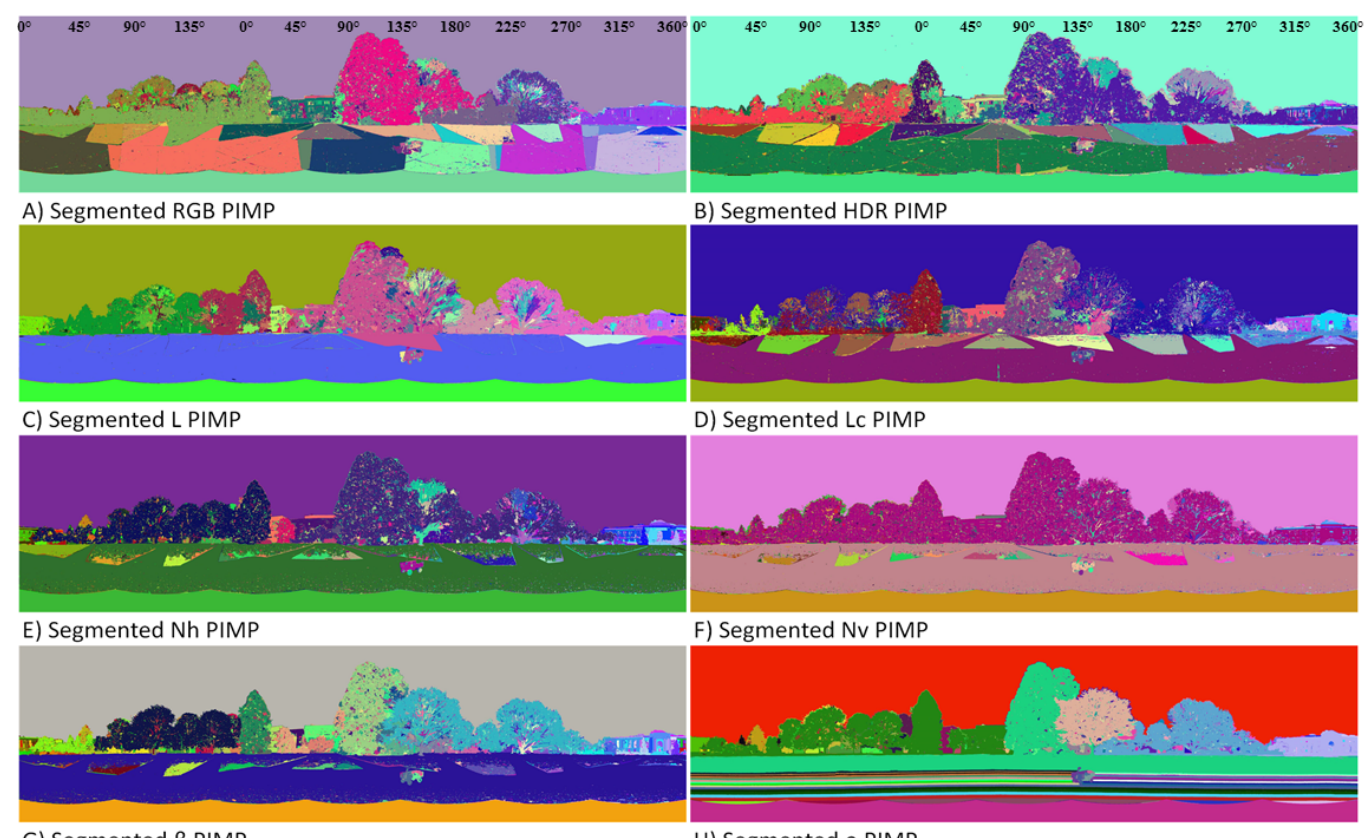

G) Segmented $\beta$ PIMP

H) Segmented $\rho$ PIMP

Figure 5: The results of graph based segmentation on generated PIMPs on the generated PIMPs for the MU dataset.

\subsection{Integration}

In the integration step, we want to blend the results of the segmentation algorithm on the different input PIMPs in order to optimize the segmentation procedure. For the terrestrial laser scanners providing data in gridded files (e.g., PTX, TZF, FLS, E57), it is possible to generate range $(\rho)$, normal vectors $\left(N_{h}, N_{v}\right)$, incidence angle $(\beta)$ and laser intensity $(L)$ PIMPs. We named these bitmaps as basic PIMPs. Further, the raw laser intensity values can be improved by applying the proposed empirical formula $\left(L_{c}\right)$. If the scanners are equipped with the digital cameras, the PIMPs of RGB bands of digital images can be created. As mentioned previously, the implementation of the HDR technique improves the RGB information by capturing several single exposure images to generate a HDR PIMP. 
To integrate the results of image segmentation algorithm on the selected PIMPs, an edge detection procedure is applied on each label matrix by taking the gradient in $\mathrm{u}$ and $\mathrm{v}$ directions and identifying the absolute values which are greater than zero. The result consists of black and white pixels showing edges of the segments on an edge PIMP (EPIMP). The union of the all boundaries is completed by stacking of all the EPIMPs, which integrates the boundary properties of desired segmented images.
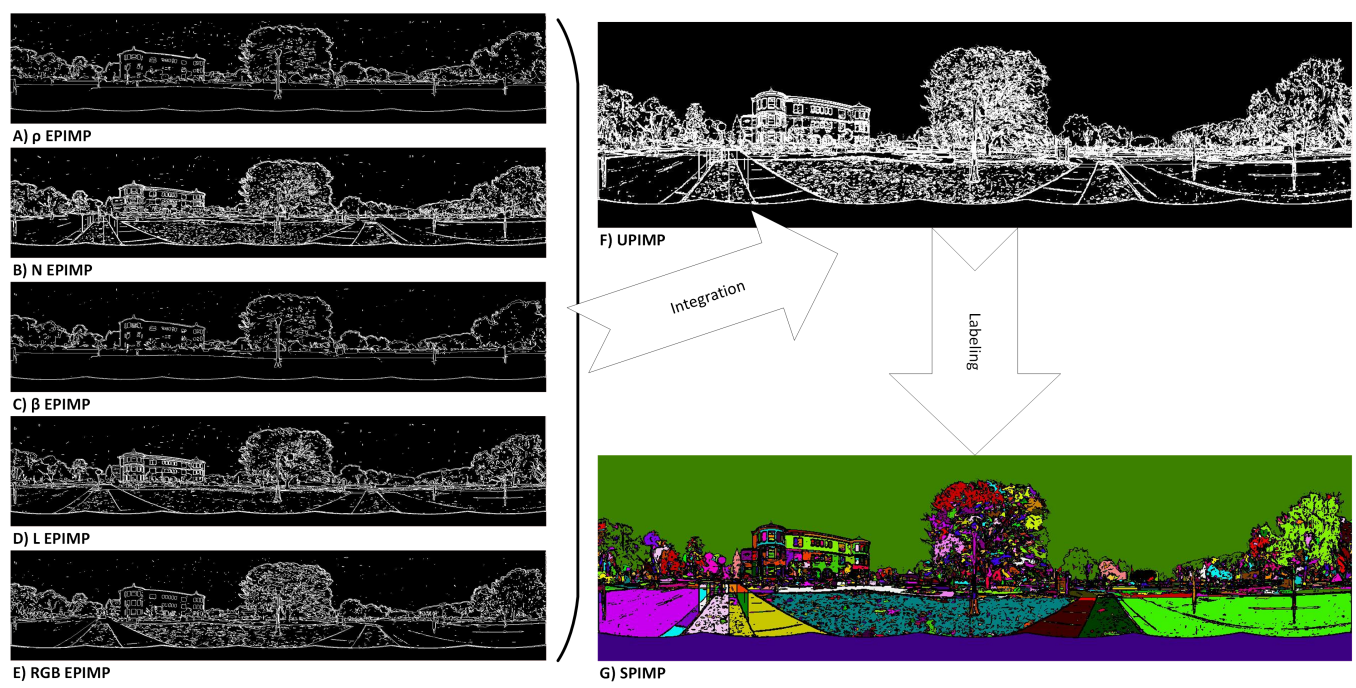

Figure 6: Integration process for the input PIMPs showing the edges of segmented images (EPIMPs) (A,B,C,D,E), union of the all boundaries (UPIMP) (F), and over-segmented label matrix $(\operatorname{SPIMP})(\mathrm{G})$

The union of all EPIMPs (UPIMP) can be treated as a probability map showing the probability of pixels representing edges. For example, a pixel with a zero value in the integrated image has the least probability of being an edge and a pixel in integrated image with the maximum value is more likely to be an edge. Pixels in the UPIMP with probability values greater than 0.5 are considered as the boarder pixels between different segments.

Applying a modified version of a connected component algorithm on the UPIMP creates an over-segmented label matrix (SPIMP). Figure 6 shows the integration procedure on an example of input PIMPs.

The neighboring labels for all clusters are found in the over-segmented label matrix (SPIMP) in a sparse matrix where each component contains the number of bordering pixels between each cluster. The value of each 
component in the sparse matrix with specific row $(i)$ and column $(j)$ indicates the number of common boundary pixels $(n)$ between segments $i$ and $j$.

\subsection{Merging}

This section describes the procedure to merge the clusters in the SPIMP based on similarity. Projecting 3D point clouds in a 360-degree terrestrial laser scanning to a $2 \mathrm{D}$ panoramic map splits the start and stop points as 0 and 360 degrees. To remove this artificial split and merge these segments, the following procedure is applied from the top pixel of the first and last column of the SPIMP to the bottom row:

- for pixels with the same segment ID, remove the border between them

- for pixels with different segment IDs, reassign the ID of the segment with the most neighboring pixels along the border to the ID of the larger segment.

To merge other (interior) segments, the first step is to describe each cluster $(i)$ based on the input PIMPs by a feature vector $(V)$ as following:

$$
V_{i}=\left[\begin{array}{ll}
\mu_{i k} & \sigma_{i k}
\end{array}\right]^{T} \quad \text { where } k=1 \ldots K
$$

where $\mu$ and $\sigma$ are the average and standard deviation values of the available PIMPs. For example, if input PIMPs are $N_{h}, N_{v}, \rho, R G B$, and $L$, the feature vector for each cluster $V_{i}$ contains 5 averages and 5 standard deviation values.

Considering the number of PIMPs $(K)$, the distance $(\delta)$ between two neighboring segments is simply calculated by a weighted Euclidean distance between their descriptor vectors as:

$$
\delta_{i, j}=\sum_{k=1}^{K} W_{k} \cdot\left\|V_{i_{k}}-V_{j_{k}}\right\|
$$

By default, the values of the weight vector are equal to one. Increasing the weight value of a parameter, places more emphasis on that parameter in order to merge the neighboring clusters. Calculating the distances between all clusters and their neighbors enables the use of an agglomerative merging algorithm. In the merging step, a user-defined threshold $(\lambda)$ is set to merge the clusters with smaller distance values (i.e., segments with similar properties). When merging two clusters, the label of the segment with fewer pixels is change to the label of the larger segment. 
In implementation, $\lambda$ can either be defined as the number of output clusters or percentage of the cluster reduction. Because this parameter is dependent of the scene, it can be easily tuned to attain the desired level of detail in the segmentation.

\section{Results and discussion}

The algorithm was tested on several TLS datasets that were acquired in different indoor and outdoor environments. All datasets feature a significant amount of occlusions and clutter, common to most TLS surveys. Table 2 provides an overview of the data and samples used in this paper.

Table 2: General attributes of the indoor and outdoor datasets selected for testing

Site ID \# of points Dimensions Description

(millions) (pixel)

1 Owen $40.1 \quad 12054 \times 3337$ Inside of Owen Hall in OSU

containing bricks, wooden doors, stairs, leather chars, etc.

2 Waldo $0.9 \quad 902 \times 1094 \quad$ Outside of Waldo Hall in OSU

containing a building, trees, asphalt, concrete, cars, close to an open porch, etc.

3 Kearney $18.5 \quad 12054 \times 3337$ Outside of Kearney Hall in OSU

an urban scene with a building, trees, stop signs, light poles, grass, concrete, etc.

In implementation, users can freely choose their preferred PIMPs. Considering the output of the proposed data improvement methods as additional PIMPs, their influence on the results could be evaluated by creating different groups of input PIMPs. However, for this research, three input data types were created for each scanned scene as shown in Table 3 as following:

- Data type I: includes basic PIMPs, which contains range, incident angle, horizontal and vertical normal vectors, laser intensity, and RGB PIMPs.

- Data type II: includes basic PIMPs, RGB, and corrected laser intensity PIMPs.

- Data type III: includes basic PIMPs, corrected laser intensity, and HDR Pimps. 
The major difference between data types I and II is the replacement of the laser intensity PIMP with corrected laser intensity values. Also data types III has both enhanced PIMPs (HDR and corrected intensity) instead of RGB and laser intensity PIMPs.

Table 3: Different groups of input PIMPs (data types) that are used to evaluate the performance of the method

\begin{tabular}{l|l|c|c|c}
\multicolumn{2}{l|}{ Input PIMP } & Data Type I & Data Type II & Data Type III \\
\hline \multirow{5}{*}{ Basic } & Range & $\checkmark$ & $\checkmark$ & $\checkmark$ \\
& Horizontal normal & $\checkmark$ & $\checkmark$ & $\checkmark$ \\
& Vertical normal & $\checkmark$ & $\checkmark$ & $\checkmark$ \\
& Incidence Angle & $\checkmark$ & $\checkmark$ & $\checkmark$ \\
\hline Laser intensity & $\checkmark$ & $\checkmark$ & $\checkmark$ \\
Corrected laser intensity & & $\checkmark$ & $\checkmark$
\end{tabular}

\subsection{Merging threshold $(\lambda)$}

As mentioned previously, the merging threshold $(\lambda)$ controls the sensitivity of the segmentation. Increasing the merging threshold decreases the number of clusters and vice versa. To illustrate the effect of different $\lambda$, basic and laser intensity PIMPs of Waldo dataset are used as input PIMPs and the results of the proposed approach using different $\lambda$ are presented in Figure 7.

The top image in Figure $7(\mathrm{~A})$ is the RGB PIMP of the scene which can be used to get an idea about the real scene. Presented images in Figure $7(\mathrm{~B}, \mathrm{C}, \mathrm{D})$ are the results of the segmentation with three different $\lambda$ as 0.1 , 0.5 , and 0.8 , respectively. Subsets of the 3D point cloud of the regions inside of the yellow boxes on each 2D panorama are shown on the right side of each 2D panorama. Because the area of interest spans the 0 and 360 degree marks (i.e., start and stop point of the scan) of the panorama, the box is divided into two sections.

Representation of the 3D point clouds of the enclosed areas in the yellow boxes are shown far right of the Figure (7). As the picture shows, the artificial split of $0^{\circ} / 360^{\circ}$ is removed and for the image with the smaller $\lambda$ value the background building is segmented into more clusters.

Figure 7(B) shows the roof and walls of the building in many segments, while $7(\mathrm{D})$ has fewer segments to show different parts of the building. The 
details of the objects behind the foreground kiosk are clearer in the segmentation with $\lambda=0.1$ and the columns of the kiosk are segmented in different parts. As a larger $\lambda$ is selected, the closer clusters are merged together and result less number of clusters. For example, using $\lambda=0.8$ merged many objects in the background of the kiosk with its columns and basically showed a few segments. So, using a smaller $\lambda$ results in more details and a larger number of small clusters (Figure $7(\mathrm{~B})$ ), while increasing $\lambda$ results in fewer, but larger clusters (Figure 7(D)).

Segmentation is a very perceptual task, meaning different people may have different needs and intents for their segmentation. For example, one may be interested to extract a large object (e.g., a building) from other objects while another wants to distinguish details of an object (e.g., windows of a building). $\lambda$ can be used to tune the segmentation to the desired level of detail.

\subsection{Improvements of laser correction and HDR}

To evaluate the effects of utilizing the proposed empirical function for laser intensity values and the HDR technique for digital images on the performance of the segmentation, three input data type are generated (Table 3). The difference between the results of data types I and II indicate the effect of applying the laser intensity correction and the results of the proposed approach on data types II and III show the influence of using HDR imaging as colorimetric data improvement.

For this portion of the analysis, the merging step was not applied on any of these test datasets since the full ability of segmentation was intended. Figures 8-12 demonstrate the results of the segmentation approach Owen and Kearney datasets. In all these figures label $A$ is the RGB PIMP or 3D view of the points colored by single shot digital images for scene visualization. Images with labels B, C, and D are the results of the proposed segmentation approach on data types I, II, and III, respectively.

Figure 8 shows the results of the proposed method on different data types of Owen dataset. Regardless of the significant amount of objects in the scene, two areas are labeled in the image as Floor and Chairs. A closer look at these areas shows that the Floor area in the Figure $8(\mathrm{C})$, which is using the corrected laser intensity PIMP, is more uniform than Figure 8(B). Figure 8(D), which is the results of data type III containing HDR PIMP, both floor pads are segmented and the Floor is segmented with more details. In the areas that are labeled as Chairs, Figure 8(B) grouped two chairs with the 


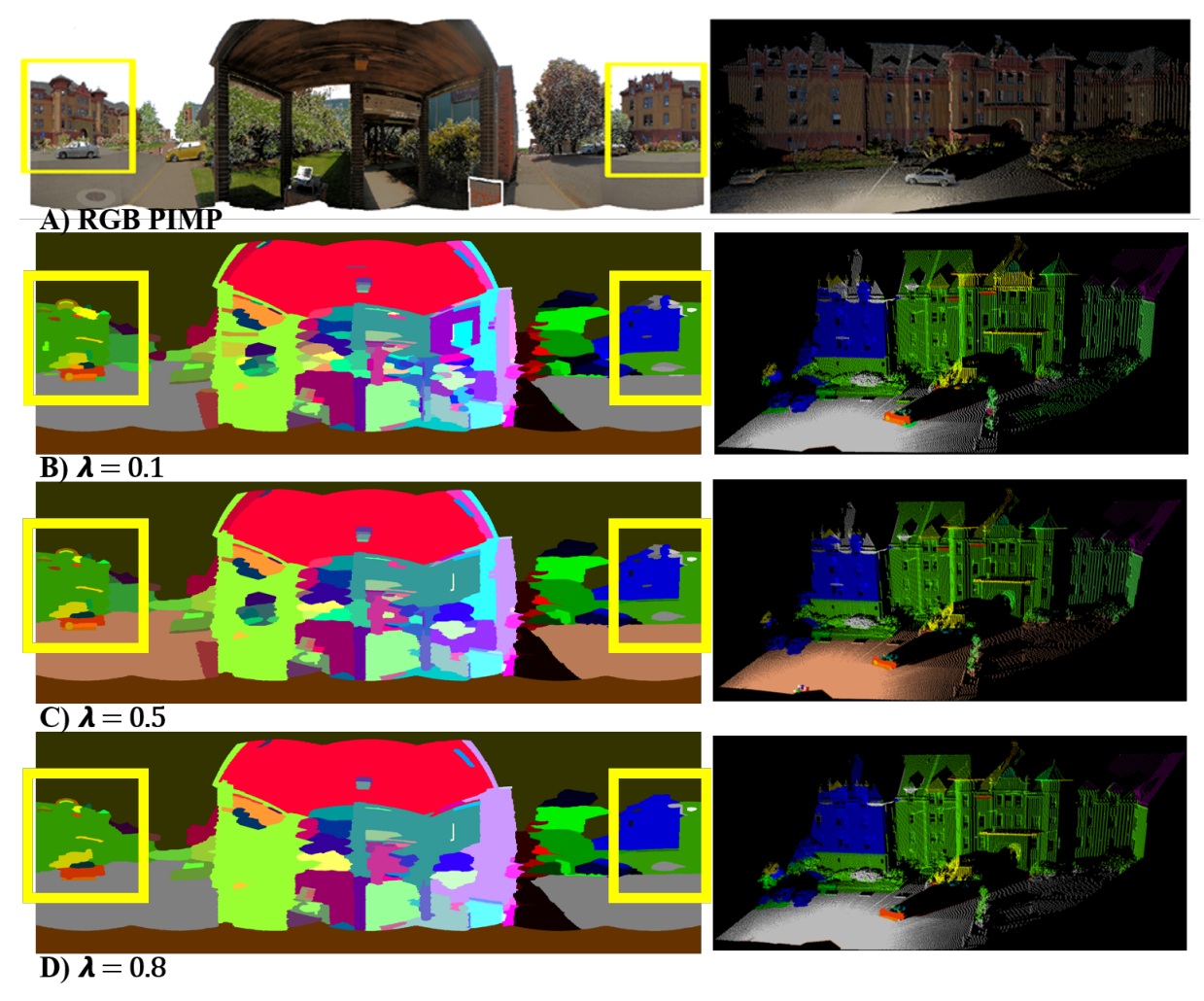

Figure 7: A) HDR PIMP of Waldo scan data with a small section highlighted in 3D, B) segmentation results with a small merging threshold $(\lambda=0.1) \mathrm{C})$ segmentation results with a medium merging threshold $(\lambda=0.5) \mathrm{D})$ segmentation results with a big merging threshold $(\lambda=0.8)$. Note that the subset image shown in 3D spans the starting $(0 \mathrm{deg})$ and end point (360 deg) of the scan.

big window in the background and Figure $8(\mathrm{C})$ grouped one chair with the background. Using both improved PIMPs (corrected intensity and HDR)in Figure 8(D) helped to segment all three chairs correctly.

The enclosed areas in black and blue boxes in Figure 8 are magnified in Figures 9 and 10 respectively. The colorized 3D points in Figure 9(A) showed the artifact caused by luminance inconsistencies between adjacent images. This inconsistencies led to false segmentation in both Figures 9(B) and (C) but it is solved in Figure 9(D) which is using data type III including HDR PIMP. These inconsistencies show up as a strong edge effect in images and finally lead to false segmentation. So using HDR PIMP is a good solution to improve the segmentation results where there are luminance inconsistencies 


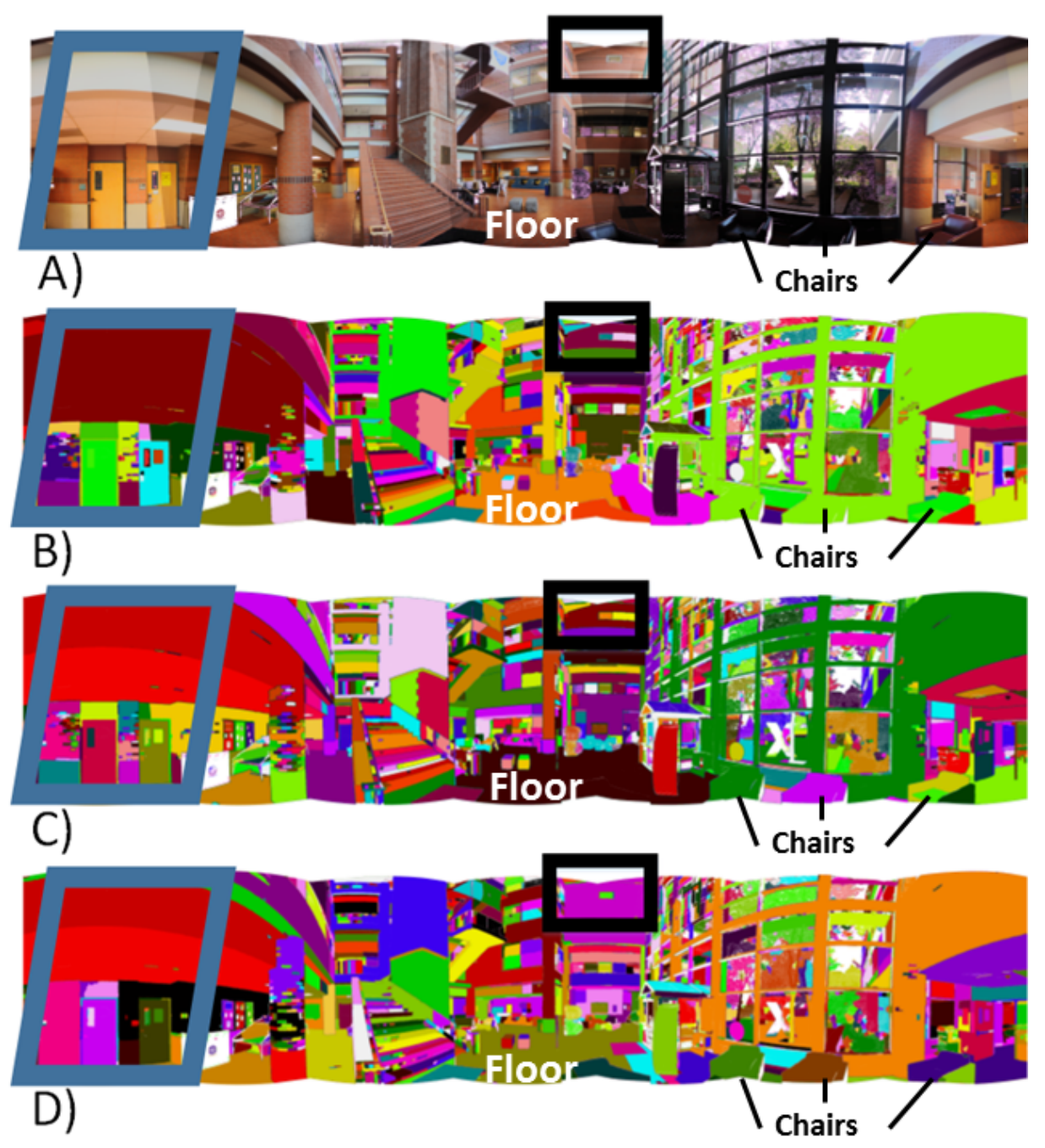

Figure 8: The results of the proposed segmentation approach on three different data types on the Owen scan scene. The areas encircled in blue and black boxes contain luminance inconsistencies. A) RGB PIMP B)segmentation results on data type I, C) segmentation results on data type II. The special PIMP for type II is corrected intensity values D) segmentation results on data type III. The special PIMPs for type III are corrected intensity and HDR. 
between adjacent images. Figure 10(A) grouped both white and brown parts of the ceiling but these parts are segmented correctly in Figures 10(C) and (D). Also, result of using data type III (Figure 10(D)) showed more uniform clusters for the brick wall.

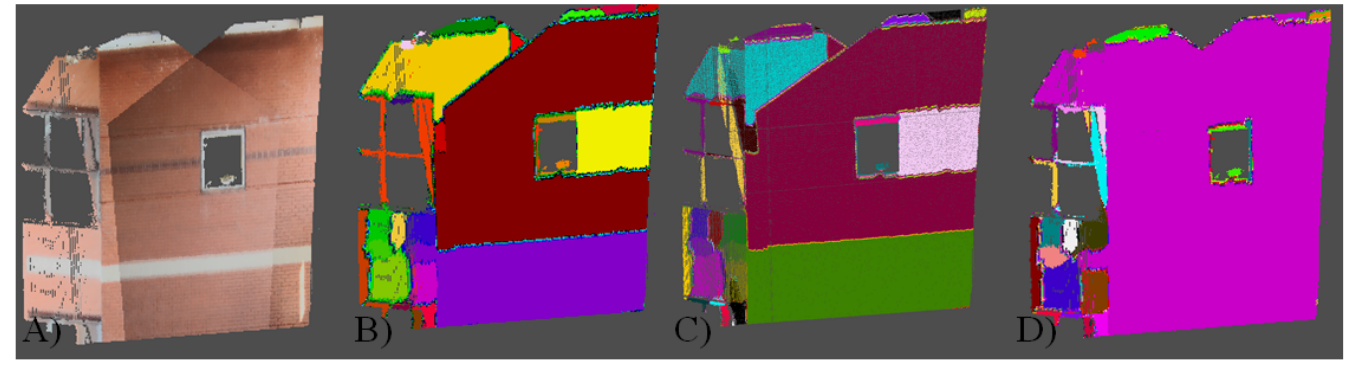

Figure 9: 3D point clouds of the enclosed area by the black box in figure 8. A) RGB colored points B) segmentation results on data type I, C) segmentation results on data type II, D) segmentation results on data type III

Figures 11 and 12 show the results of the proposed approach on Kearney dataset. The segmentation approach on data type II provides the ability to distinguish more clusters compared to data type I. For example, the extraction of the sign and parts of the pavements is more successful. Using corrected laser intensities groups the building sections together while also separating trees into different clusters because of the increased variability in values. Applying both improvement techniques (data type III) results in the best segmentation for the walkways, stop signs, power poles and building.

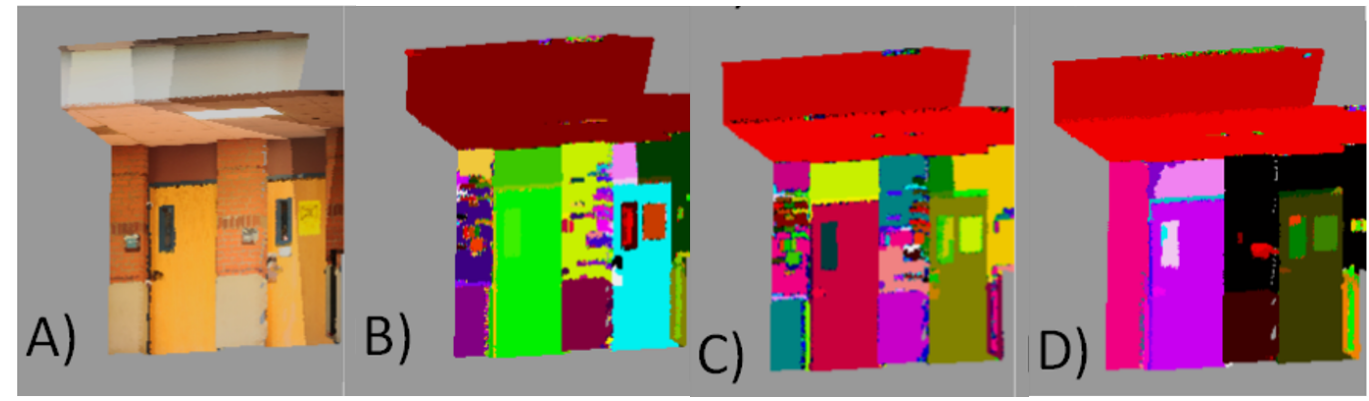

Figure 10: 3D point clouds of the enclosed area by the blue parallelogram in figure 8 . A) RGB colored points B) segmentation results on data type I, C) segmentation results on data type II, D) segmentation results on data type III 


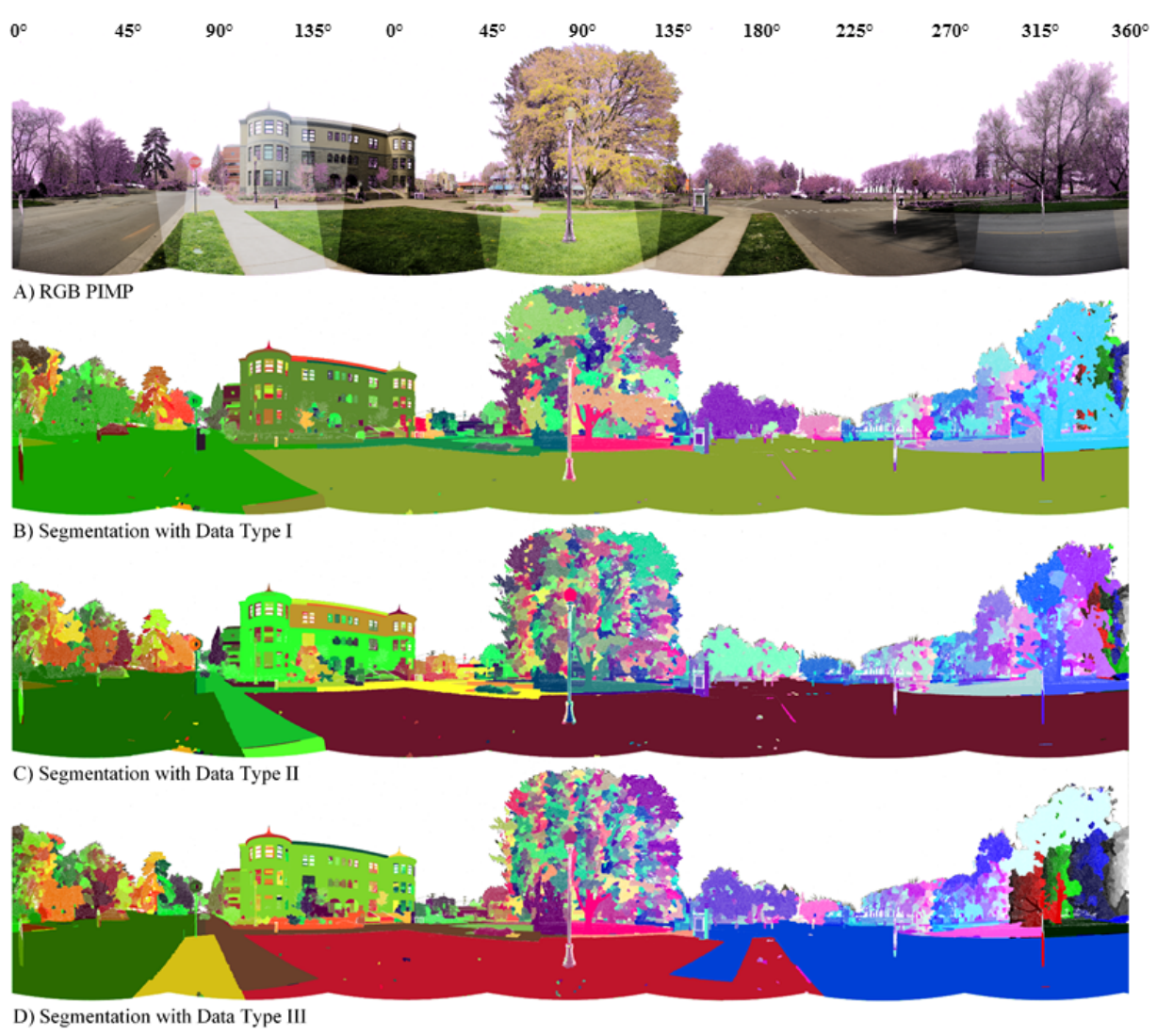

Figure 11: A) RGB PIMP for the Kearney scan scene. The influence of data improvement techniques on the Kearney scan scene are shown for data types I (B), II (C), and III (D).

The general improvement was using HDR improvement reduce the false segmentations because of the luminance inconsistencies in the scene. Also using laser intensity corrected values results more uniform segmentation for the objects with the same materials. In general, for all scans, by applying both proposed data improvement techniques (data type III), the segmentation approach grouped the points from the same objects together with less false segmentation.

\subsection{Comparison to RANSAC algorithm}

To evaluate the performance of the proposed segmentation, it is compared with the state-of-the-art RANSAC point cloud segmentation in an open source package, CloudCompare (Giraud-Monte, 2013). An automatic 


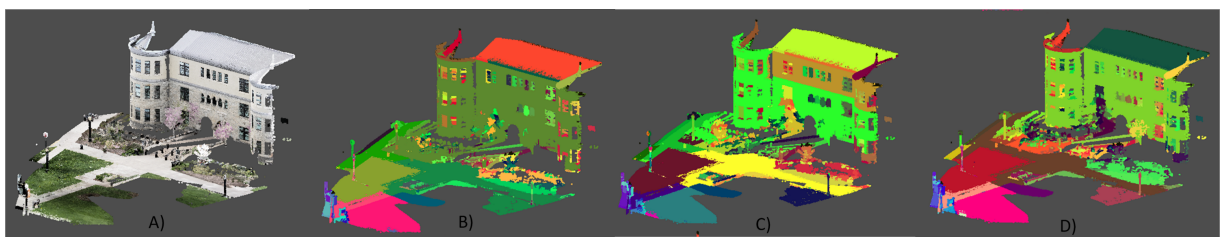

Figure 12: 3D point clouds of the Kearney scan scene and the results of the segmentation on its different data type. A) colored points B) segmentation on data type I, C) segmentation on data type II, D) segmentation on data type III.

shape detection algorithm proposed by (Schnabel et al., 2007) is implemented in the software as "RANSAC Shape Detection". The plugin is able to detect five types of primitives: planes, spheres, cylinders, cones, and tori.

Comparisons are made based on the execution time and qualitative results. The subsampling ratio to reduce the image size clearly has a significant influence on execution time, with minimal effect on the results. The execution times for the Owen Hall scan scene with four different subsampling ratio are measured as shown in Table 4. To match this level of reduction when segmenting with the RANSAC algorithm, the RANSAC algorithm was applied to the same point cloud (Owen Hall) with different level of point cloud decimations reducing it to the same number of samples. The comparison of the execution times between the proposed approach which is applied to 3D data in a gridded structure and RANSAC that works on 3D point clouds after basic spatial structuring (e.g., octree) is evaluated. This comparison shows the benefits of utilizing the gridded structure and image processing algorithms over 3D geometric methods.

Table 4 shows that the proposed approach runs significantly faster than RANSAC for the dense datasets. When the datasets are significantly reduced in size, the performance of both approaches are similar. As briefly mentioned in Section 2, the existing lidar point cloud segmentation techniques are not equipped to handle large datasets (several to tens of millions of points) whereas Table 4 shows that the proposed approach works well for these large datasets, while computation time increases exponentially for RANSAC.

The qualitative results of RANSAC on the Kearney scan is presented in Figure 13. Because the points only fit on a very small portion of the complete primitive, usually these primitives are much larger than the actual cloud. 
Table 4: Comparison of the execution time between the proposed method and RANSAC for the Owen Hall dataset. The computer that was used for the processing had 48 GB RAM, Intel(R) Xeon(R) CPU E5620 @ 2.40GHz, running on 64-bit Windows 7.

\begin{tabular}{l|c|c|c|c} 
Subsampling ratio & 1 & 0.75 & 0.5 & 0.25 \\
\hline Image size (pixels) & $12053 \times 3337$ & $9040 \times 2503$ & $6027 \times 1669$ & $3014 \times 835$ \\
\# of points (Million) & 40.1 & 30.1 & 20.0 & 10.0 \\
Proposed method time (sec) & 1555 & 713 & 256 & 160 \\
RANSAC time (sec) & 6005 & 5406 & 1460 & 300
\end{tabular}

Note that in these figures, each segment of the point cloud is designated by a distinct color that is randomly assigned.

Figure 13 shows the qualitative comparison of the segmentation results applying the proposed algorithm and RANSAC. Figure 13(A) shows the results of the proposed approach on a part of scan data, while the Figure 13(B) is the same scene segmented by RANSAC. The color fluctuations present in the RANSAC results indicate that it cannot properly segment objects with complex shapes. On the other hand, the proposed approach successfully grouped different patches of the ground, light pole, trunk and leaves of the trees.

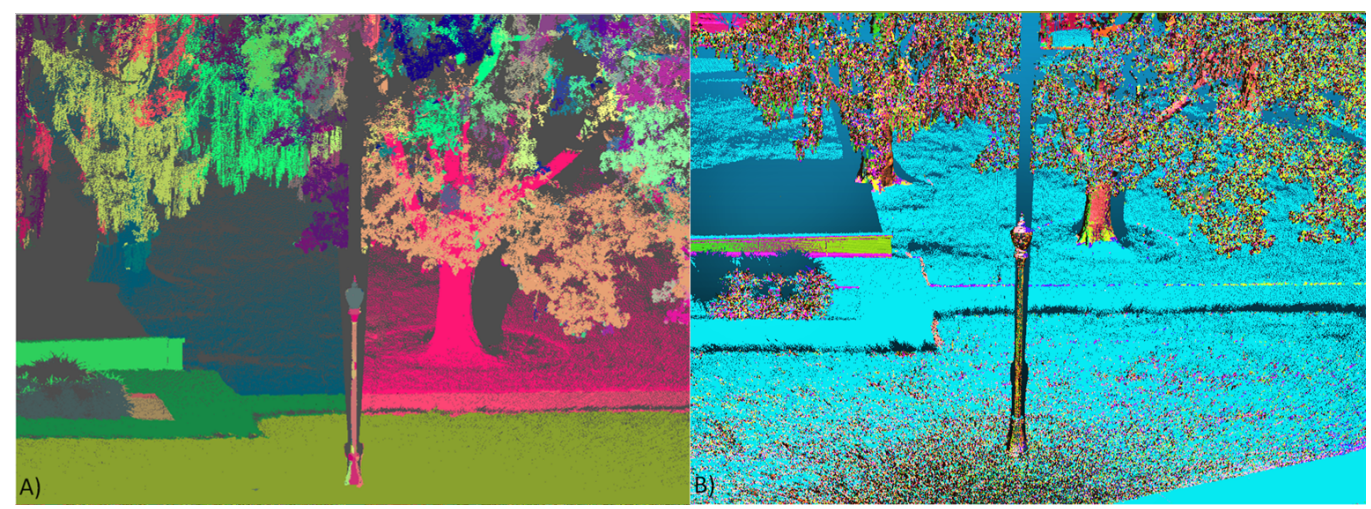

Figure 13: Qualitative comparison between the results of A) the proposed approach and B) RANSAC on the same scene.

\subsection{Quantitative validation}

Commonly, the performance of segmentation is evaluated by the reader's intuition and perception. Quantification, particularly in complex scenes, is 
difficult because of challenges in manually segmenting the point cloud beyond a certain level of detail. While the previous examples show the effectiveness of the algorithm on complex, cluttered scenes, this section will quantitatively evaluate the performance on a relatively simple scene that could be reasonably segmented manually. This classroom has well defined ceiling tiles, more or less bare walls, a desk, door, trashcan, and white board (Figure 14).

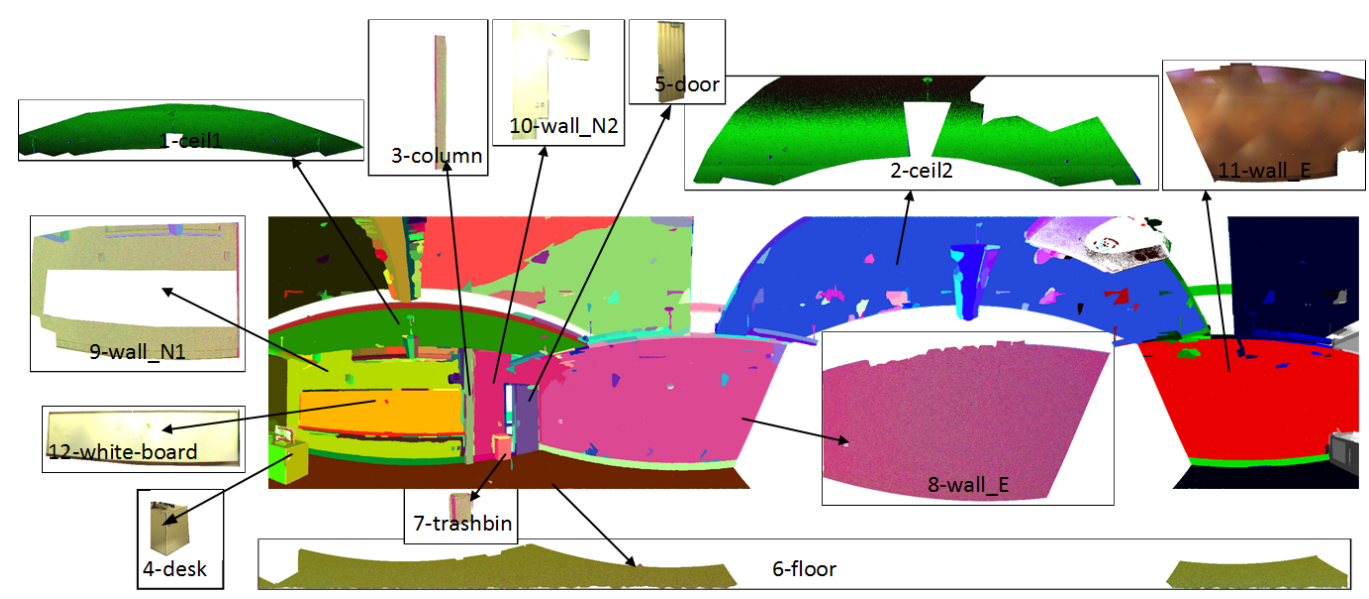

Figure 14: The RGB PIMP of a simple scan scene (classroom) and its selected semantic objects.

A ground-truth segmentation was obtained by manually segmenting the RGB PIMP for each of the aforementioned objects. The results of the segmentation using the proposed algorithm were then compared to this manual segmentation pixel to pixel. In addition to calculate the widely used information retrieval measurements such as recall, precision, and F-measure, the Matthew Correlation Coefficient (MCC) is also computed (Powers, 2011). MCC is generally regarded as a balanced measure which can be used even if the classes are of very different sizes. It returns a value between -1 and +1 where a coefficient of +1 represents a perfect prediction, 0 no better than random prediction and -1 indicates total disagreement between prediction and observation (Powers, 2011). Calculating true positive (TP), true negative $(\mathrm{TN})$, false positive (FP), false negative $(\mathrm{FN})$ from the confusion matrix leads to compute MCC with the following formula:

$$
M C C=\frac{T P \times T N-F P \times F N}{\sqrt{(T P+F P)(T P+F N)(T N+F P)(T N+F N)}}
$$


Figure 14 and Table 5 show the selected objects and resulting quantitative results, respectively. The average of MCC values for all objects in the scene is 0.87 which shows the proposed approach is a reliable method for point cloud segmentation. However, it is worth mentioning the manual segmentation grossly oversimplifies the scene and ignores smaller objects like the speakers on the wall, eraser on the chalkboard, electrical outlets, etc. that were segmented using our approach. Hence, if a more detailed manual segmentation were performed, the MCC values would be even higher.

Table 5: The quantitative evaluation of the segmentation approach on a simple scan scene

\begin{tabular}{l|cccc} 
& MCC & Recall & Precision & F-measure \\
\hline Ceiling 1 & 0.963 & 0.945 & 0.985 & 0.964 \\
Ceiling 2 & 0.935 & 0.905 & 0.981 & 0.942 \\
Column & 0.761 & 0.581 & 1.000 & 0.735 \\
Desk & 0.874 & 0.782 & 0.978 & 0.869 \\
Door & 0.905 & 0.820 & 0.999 & 0.901 \\
Floor & 0.960 & 0.954 & 0.970 & 0.962 \\
Trashbin & 0.681 & 0.464 & 1.000 & 0.634 \\
Wall - E & 0.913 & 0.850 & 0.995 & 0.917 \\
Wall - N1 & 0.763 & 0.596 & 0.993 & 0.745 \\
Wall - N2 & 0.851 & 0.868 & 0.838 & 0.853 \\
Wall - W & 0.940 & 0.893 & 0.999 & 0.943 \\
Whiteboard & 0.904 & 0.821 & 1.000 & 0.901
\end{tabular}

\section{Conclusions}

Exploiting advanced HDR photography, intensity normalization, and available computer vision image segmentation algorithms improves lidar point cloud segmentation and visualization. This research addressed three main objectives: 1) develop a segmentation approach by applying computer vision algorithms, 2) implement the HDR photography to improve colorimetric data and consequently segmentation results, 3) improve the performance of the segmentation by utilizing an empirical correction formula to correct laser intensity values. Herein, we will describe how each objective was fulfilled.

1) Develop an efficient, fast, comprehensive point cloud segmentation approach by applying computer vision algorithms

In this research, a novel approach is introduced to apply computer vision 
algorithms for point cloud data processing. Using the PIMP representation of a scan simplified data manipulation and primitive segmentation compared to directly processing the 3D point cloud datasets. Minimal computing power is necessary to work with $2 \mathrm{D}$ images compared to the resources needed to display and manipulate massive 3D point cloud datasets. Further, the PIMP form enables one to utilize computer vision and other image processing techniques for TLS point cloud processing.

Although different people may intend different level of segmentation, which makes the evaluation of the segmentation methods challenging, the proposed approach showed good agreement with a manual classification $(0.87$ average MCC). The proposed approach is tested on several lidar datasets and compared with state of art RANSAC method available in CloudCompare software. The comparison showed reduction in computation time, qualitative improvement in point cloud segmentation, and minimization of user intervention.

2) Implement HDR photography to improve digital images and consequently segmentation result

Enhancing point cloud visualization and segmentation using HDR asserts that implementation of HDR technique within the data capture process and subsequent processing workflow should represent the next significant development within terrestrial laser scanning and could give significant improvements to the colorimetric data. Using the HDR PIMP in segmentation

minimize artifacts due to luminance inconsistencies between adjacent images in areas of overlapping images.

3) Improve the performance of the segmentation by utilizing an empirical correction formula to correct laser intensity values for range and incidence angle

An empirical correction function is introduced to modify the observed laser intensities to alleviate the effect of range (distance) and incidence angle (target surface properties). Using the PIMP of corrected laser intensities as an input in this method groups parts of solid objects such as ground and buildings while splitting objects with various ranges of laser intensities such as trees and grass.

Unlike segmentation approaches that work directly on 3D point clouds, this approach can only work with one scan at a time. The benefit of working with a single scan is that the segmented objects could be used for registration. However, the trade-off is that there will be some oversegmentation due 
to data gaps and missing information that would be captured in another scan. Another limitation is that the approach requires TLS datasets with an angular structure format so it cannot be applied to generic point clouds such as those derived from structure from motion. However, in those cases, could create a gridded point cloud from an unstructured point cloud via virtual scanning. The authors hope to tackle these limitations in future work.

\section{Acknowledgments}

This material is based upon work supported by the National Science Foundation under Grant No. 1351487.

\section{References}

Barnea, S., Filin, S., 2008a. Segmentation of terrestrial laser scanning data by integrating range and image content. In: The Proceedings of XXIth ISPRS Congress. Citeseer.

Barnea, S., Filin, S., 2008b. Segmentation of terrestrial laser scanning data by integrating range and image content. In: The Proceedings of XXIth ISPRS Congress. Citeseer.

Barnea, S., Filin, S., 2012. Extraction of objects from terrestrial laser scans by integrating geometry image and intensity data with demonstration on trees. Remote Sensing 4 (1), 88-110.

Barnea, S., Filin, S., Alchanatis, V., 2007. A supervised approach for object extraction from terrestrial laser point clouds demonstrated on trees. PIA07-Photogrammetric Image Analysis, The International Archives of the Photogrammetry, Remote Sensing and Spatial Information Science 36, W49A.

Biosca, J. M., Lerma, J. L., 2008. Unsupervised robust planar segmentation of terrestrial laser scanner point clouds based on fuzzy clustering methods. ISPRS Journal of Photogrammetry and Remote Sensing 63 (1), 84-98.

Briese, C., Pfennigbauer, M., Lehner, H., Ullrich, A., Wagner, W., Pfeifer, N., 2012. Radiometric calibration of multi-wavelength airborne laser scanning data. ISPRS Ann. Photogramm. Remote Sens. Spat. Inf. Sci 1, 335-340. 
Caroti, G., Piemonte, A., 2007. Laser scanner and photogrammetry for the survey of the monumental cemetery in piazza del duomo, pisa (italy). Vol. 36. p. C53.

Chehata, N., David, N., Bretar, F., 2008. Lidar data classification using hierarchical k-means clustering. In: ISPRS Congress Beijing 2008. Vol. 37. Citeseer, pp. 325-330.

Cheng, L., Gong, J., Li, M., Liu, Y., 2011. 3d building model reconstruction from multi-view aerial imagery and lidar data. Photogrammetric Engineering \& Remote Sensing 77 (2), 125-139.

Dallal, G. V., et al., 1999. The little handbook of statistical practice. Gerard V. Dallal.

Ding, Q., Chen, W., King, B., Liu, Y., Liu, G., 2013. Combination of overlapdriven adjustment and phong model for lidar intensity correction. ISPRS Journal of Photogrammetry and Remote Sensing 75, 40-47.

Douillard, B., Underwood, J., Kuntz, N., Vlaskine, V., Quadros, A., Morton, P., Frenkel, A., 2011. On the segmentation of 3d lidar point clouds. In: Robotics and Automation (ICRA), 2011 IEEE International Conference on. IEEE, pp. 2798-2805.

Felzenszwalb, P. F., Huttenlocher, D. P., 2004. Efficient graph-based image segmentation. International Journal of Computer Vision 59 (2), 167-181.

Fischler, M. A., Bolles, R. C., 1981. Random sample consensus: a paradigm for model fitting with applications to image analysis and automated cartography. Communications of the ACM 24 (6), 381-395.

Giraud-Monte, D., 2013. CloudCompare User's Manual for version 2.1. URL www.cloudcompare.net

Gorte, B., 2007. Planar feature extraction in terrestrial laser scans using gradient based range image segmentation. In: ISPRS Workshop on Laser Scanning. pp. 173-177.

Höfle, B., Pfeifer, N., 2007. Correction of laser scanning intensity data: Data and model-driven approaches. ISPRS Journal of Photogrammetry and Remote Sensing 62 (6), 415-433. 
Huber, D., 2011. A new, open standard for 3d imaging data.

Jutzi, B., Gross, H., 2009. Normalization of lidar intensity data based on range and surface incidence angle. Int. Arch. Photogramm. Remote Sens. Spat. Inf. Sci 38, 213-218.

Kaasalainen, S., Jaakkola, A., Kaasalainen, M., Krooks, A., Kukko, A., 2011. Analysis of incidence angle and distance effects on terrestrial laser scanner intensity: search for correction methods. Remote Sensing 3 (10), 22072221.

Kaasalainen, S., Krooks, A., Kukko, A., Kaartinen, H., 2009. Radiometric calibration of terrestrial laser scanners with external reference targets. Remote Sensing 1 (3), 144-158.

Kadobayashi, R., Kochi, N., Otani, H., Furukawa, R., 2004. Comparison and evaluation of laser scanning and photogrammetry and their combined use for digital recording of cultural heritage. International Archives of the Photogrammetry, Remote Sensing and Spatial Information Sciences 35 (5), 401-406.

Kashani, A. G., Olsen, M. J., Parrish, C. E., Wilson, N., 2015. A review of lidar radiometric processing: From ad hoc intensity correction to rigorous radiometric calibration. Sensors 15 (11), 28099-28128.

Leica, G., 2014. Leica Cyclone 8.0 Help.

Lin, C.-C., You, R.-J., 2006. Planar feature extraction from lidar data based on tensot analysis. pp. 1-7.

Mahmoudabadi, H., 2015. Implementing high dynamic range (hdr) photography to improve $3 \mathrm{~d}$ laser scanning point cloud visualization and segmentation. Phd dissertation, School of Civil and Construction Engineering, Oregon State University.

Mahmoudabadi, H., Shoaf, T., Olsen, M. J., 2013. Superpixel clustering and planar fit segmentation of 3d lidar point clouds. In: Computing for Geospatial Research and Application (COM. Geo), 2013 Fourth International Conference on. IEEE, pp. 1-7. 
Mallet, C., Bretar, F., Roux, M., Soergel, U., Heipke, C., 2011. Relevance assessment of full-waveform lidar data for urban area classification. ISPRS Journal of Photogrammetry and Remote Sensing 66 (6), S71-S84.

Moussa, A., El-Sheimy, N., 2010. Automatic classification and 3d modeling of lidar data. In: Proceedings of the ISPRS Commission III symposium-PCV 2010 .

Niemeyer, J., Rottensteiner, F., Soergel, U., 2012. Conditional random fields for lidar point cloud classification in complex urban areas. ISPRS annals of the photogrammetry, remote sensing and spatial information sciences 1 (3), 263-268.

Olsen, M., Ponto, K., Kimball, J., Seracini, M., Kuester, F., 2010. 2d opensource editing techniques for $3 d$ laser scans. Proc., Computer Applications and Quantitative Methods in ArchaeologyCAA, 47-50.

Pfeifer, N., Höfle, B., Briese, C., Rutzinger, M., Haring, A., 2008. Analysis of the backscattered energy in terrestrial laser scanning data. Int. Arch. Photogramm. Remote Sens. Spat. Inf. Sci 37, 1045-1052.

Powers, D., 2011. Evaluation: From precision, recall and f-measure to roc., informedness, markedness \& correlation. Journal of Machine Learning Technologies $2(1), 37-63$.

$\mathrm{Pu}$, S., Vosselman, G., et al., 2006. Automatic extraction of building features from terrestrial laser scanning. Vol. 36. pp. 25-27.

Rabbani, T., van den Heuvel, F., Vosselmann, G., 2006. Segmentation of point clouds using smoothness constraint. International Archives of Photogrammetry, Remote Sensing and Spatial Information Sciences 36 (5), 248-253.

Reinhard, E., Heidrich, W., Debevec, P., Pattanaik, S., Ward, G., Myszkowski, K., 2010. High dynamic range imaging: acquisition, display, and image-based lighting. Morgan Kaufmann.

Riegl, 2009. 3d terrestrial laser scanner, riegl vz-400, general description and data interfaces. 
Rusu, R. B., Cousins, S., 2011. 3d is here: Point cloud library (pcl). In: Robotics and Automation (ICRA), 2011 IEEE International Conference on. IEEE, pp. 1-4.

Schnabel, R., Wahl, R., Klein, R., 2007. Efficient ransac for point-cloud shape detection. In: Computer graphics forum. Vol. 26. Wiley Online Library, pp. $214-226$.

Serna, A., Marcotegui, B., 2014. Detection, segmentation and classification of $3 \mathrm{~d}$ urban objects using mathematical morphology and supervised learning. ISPRS Journal of Photogrammetry and Remote Sensing 93, 243-255.

Shan, J., Toth, C. K., 2008. Topographic laser ranging and scanning: principles and processing. CRC press.

Sok, C., Adams, M. D., 2010. Visually aided feature extraction from 3d range data. In: Robotics and Automation (ICRA), 2010 IEEE International Conference on. IEEE, pp. 2273-2279.

Strom, J., Richardson, A., Olson, E., 2010. Graph-based segmentation for colored 3d laser point clouds. In: Intelligent Robots and Systems (IROS), 2010 IEEE/RSJ International Conference on. IEEE, pp. 2131-2136.

Szeliski, R., 2010. Computer vision: algorithms and applications. Springer Science \& Business Media.

Triebel, R., Burgard, W., Dellaert, F., 2005. Using hierarchical em to extract planes from 3d range scans. In: Robotics and Automation, 2005. ICRA 2005. Proceedings of the 2005 IEEE International Conference on. IEEE, pp. 4437-4442.

Vain, A., Kaasalainen, S., Pyysalo, U., Krooks, A., Litkey, P., 2009. Use of naturally available reference targets to calibrate airborne laser scanning intensity data. Sensors 9 (4), 2780-2796.

Vosselman, G. V., Maas, H.-G., 2010. Airborne and terrestrial laser scanning. Whittles.

Wang, J., Shan, J., 2009. Segmentation of lidar point clouds for building extraction. In: American Society for Photogramm. Remote Sens. Annual Conference, Baltimore, MD. pp. 9-13. 
Weinmann, M., Jutzi, B., Hinz, S., Mallet, C., 2015. Semantic point cloud interpretation based on optimal neighborhoods, relevant features and efficient classifiers. ISPRS Journal of Photogrammetry and Remote Sensing 105, 286-304.

Yu, Z., Xu, C., Liu, J., Au, O. C., Tang, X., 2011. Automatic object segmentation from large scale $3 \mathrm{~d}$ urban point clouds through manifold embedded mode seeking. In: Proceedings of the 19th ACM international conference on Multimedia. ACM, pp. 1297-1300.

Zeibak, R., Filin, S., 2009. Object extraction from terrestrial laser scanning data. In: FIG Working Week. 


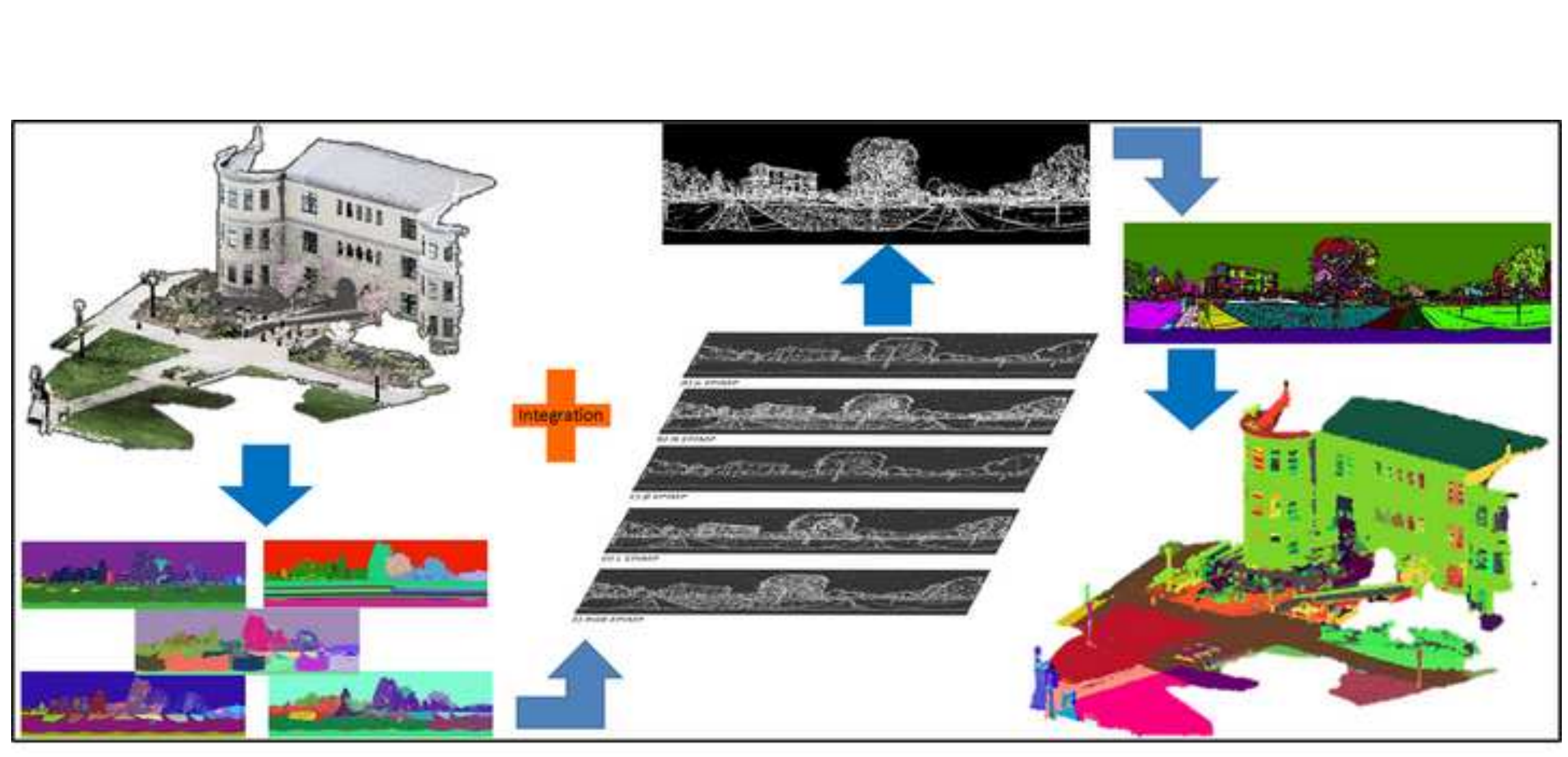

.

\author{
Graphical Absta
}

